\title{
Topological Sensitivity for Solving Inverse Multiple Scattering Problems in Three-dimensional Electromagnetism. Part I: One Step Method*
}

\author{
Frédérique Le Louër ${ }^{\dagger}$ and María-Luisa Rapún $¥$
}

\begin{abstract}
In this paper we compute closed-form expressions for the topological derivative for three-dimensional time-harmonic electromagnetic waves for perfect conductors (Dirichlet condition), electromagnetic cavities (Neumann condition), absorbing obstacles (impedance condition), and dielectric inclusions (transmission conditions). The proofs are based on the computation of shape derivatives followed by asymptotic expansions using Mie series when infinitesimal spheres are considered. An exhaustive gallery of numerical experiments is presented, which demonstrate that the topological derivative is a very powerful tool for the detection of multiple electromagnetic scatterers without a priori information about their number, size, shape, or location. Numerical examples include highly demanding configurations where only a few incident directions and a few observation points (for near-field data) or a few far-field observation directions (for far-field data) are considered.
\end{abstract}

Key words. electromagnetism, topological derivative, inverse obstacle scattering problems, shape reconstruction

AMS subject classifications. 35R30, 49Q12, 65N21, 65K10, 78A25, 78A40, 78A46

DOI. $10.1137 / 17 \mathrm{M} 1113850$

1. Introduction. Numerous physical applications such as nondestructive testing, geophysical exploration, or radar and biomedical imaging lead to the solution of inverse multiple scattering problems. As a result, the interest of the scientific community in solving inverse problems has grown significantly in the last two decades. Various new techniques have been developed in the special case of the location and shape identification problem of scatterers from far-field or near-field data. Most attention has been devoted to the following two categories of approaches: (i) nonlinear optimization methods, which iteratively recover extremely accurate approximate solutions from incomplete radiation pattern data but usually require some initial information on the scattering object; (ii) qualitative methods, which provide fast reconstruction and localization with limited initial information on the scattering object but usually require a large amount of data. Topological derivative-based methods belong to both of the two categories and overcome the main drawbacks mentioned above; namely, no a priori information about the number, size, or location of the defects is required, and furthermore, they provide very reasonable reconstructions when only a small amount of data is available.

\footnotetext{
${ }^{*}$ Received by the editors January 27, 2017; accepted for publication (in revised form) May 8, 2017; published electronically August 17, 2017.

http://www.siam.org/journals/siims/10-3/M111385.html

Funding: The work of the second author was supported by MINECO grants TRA2016-75075-R and MTM201456948-C2-1-P.

${ }^{\dagger}$ LMAC EA2222, Laboratoire de Mathématiques Appliquées de Compiègne, Sorbonne Université, Université de Technologie de Compiègne, 60203 Compiègne cedex, France (frederique.le-louer@utc.fr).

${ }^{\ddagger}$ Departamento de Matemática Aplicada a la Ingeniería Aeroespacial, ETSI Aeronáutica y del Espacio, Universidad Politécnica de Madrid, 28040 Madrid, Spain (marialuisa.rapun@upm.es).
} 
In addition, topological derivative methods are very robust with respect to noise, being able to provide accurate reconstructions even in the case of highly noisy data.

The topological derivative of a shape functional measures the sensitivity of such a functional to having an infinitesimal scatterer at each point of the region of interest. It can be used as an indicator function that classifies each point as belonging either to one scatterer or to the background media. In this paper, we study the performance of this derivative for the three-dimensional (3D) time-harmonic Maxwell equations in unbounded media for perfect conductors, electromagnetic cavities, absorbing obstacles, and dielectric inclusions (namely, when Dirichlet, Neumann, impedance, and transmission boundary conditions, respectively, are imposed on the boundary of the scatterers).

Asymptotic formulae for time-harmonic Maxwell equations in a bounded domain were derived in [3] and used in [40] to find the topological gradient in a bounded region when transmission conditions are imposed on the boundary of an infinitesimal sphere. However, numerical experiments for the derived topological derivative were not shown. From a theoretical point of view, full 3D Maxwell equations are also considered in [52, 53]. In [52], electromagnetic small inclusions are sought in a bounded domain from boundary measurements of the tangential component of the scattered magnetic field by using a filtered topological derivative, where the classical $L^{2}$-cost functional is replaced by a new functional defined in terms of boundary integral operators of Neumann-Poincaré type. In the very recent paper [53], topological derivatives are derived for electromagnetic inhomogeneities with permittivity and/or permeability contrast for the classical $L^{2}$-cost functional from measurements of the far-field amplitude of the scattering electric field. The emphasis in this paper is on theoretical results about resolution analysis and statistical stability with respect to noise. A very few numerical experiments dealing with the reconstruction of a small sphere with only permeability or permittivity contrast are presented.

To the best of our knowledge, except for the above-mentioned paper [53], the present paper is the first where numerical experiments illustrating the performance of topological derivative imaging functionals for the full Maxwell equations are provided. However, for related electromagnetic contexts it worth mentioning [40], where experiments for a 2D problem related to the detection of metallic objects buried in a solid (in the spirit of [48]), or 3D objects in free space for time-domain scattered field data, are shown. Dealing also with Helmholtz equations, 2D thin electromagnetic inclusions and cracks are considered in [45, 46], while for acoustic problems in two and three dimensions we refer the reader to $[11,12,18]$ and $[24]$, respectively (see also the recent work [10] for the application to visualization of 3D cells using light microscopes). In [2] the topological derivatives for two different cost functionals are compared for the Helmholtz case.

As mentioned above, the topological derivative provides an indicator function that classifies each point of the observed region as belonging either to the background media or to an object. This kind of method has been profusely studied in recent years. In particular, for electromagnetic scattering it is worth mentioning the papers $[8,9,13]$ for the linear sampling method, [32] for the factorization method, [4] for a multiple signal classification (MUSIC) algorithm, $[6,50]$ for the point-source method, and [23] for a comparison of sampling methods. In comparison with other qualitative methods, we will show that the topological derivative is highly competitive, since it provides very reasonable reconstructions even with a limited 
amount of noisy data, and the computational cost is almost negligible (it only requires evaluating fundamental solutions to the Maxwell system at a grid of points).

Approximations provided by the computation of the topological derivative can be used as initial guesses for iterative methods. For instance, they can be used in Newton-type algorithms [14, 27, 30, 31, 35], level set methods [16, 17], and algorithms based on the computation of iterated topological derivatives [12] and iterated topological energies [10]. In a forthcoming paper [36] we will derive the corresponding formulae for the topological derivative for implementing the iterative method proposed in [12], and test its performance numerically. The extension for the iterative computation of topological derivatives is not immediate from the results presented in this paper.

The paper is organized as follows: In section 2 we formulate the direct and inverse scattering problems for several kinds of boundary conditions in $\mathbb{R}^{3}$. The topological derivative formula can be reinterpreted using shape derivative tools [51]. Characterizations of the shape derivatives of the solution to electromagnetic scattering problems have already been established [15, 26, 29, 33, 49]. Using these results and the Mie series theory, we derive in section 3 the topological derivative of the least-squares cost functional. We present a large gallery of numerical experiments in section 4 for the Neumann boundary condition, which include one or several scatterers with nontrivial shapes and several wavenumbers. Examples dealing with limited aperture problems, different number of incident directions, and/or observation points, noisy data, far-field data, and different boundary conditions are presented in the supplementary material file M111385_01.pdf [local/web 4.66MB], which complements this section. Finally, we give concluding remarks and discuss possible research lines in section 5 .

Notation. For a given bounded domain $\Omega \subset \mathbb{R}^{3}$ we denote by $H^{s}(\Omega), H_{\text {loc }}^{s}\left(\mathbb{R}^{3} \backslash \bar{\Omega}\right)$, and $H^{s}(\partial \Omega)$ the standard (local in the case of the exterior domain) complex-valued, HilbertSobolev space of order $s \in \mathbb{R}$ defined on $\Omega, \mathbb{R}^{3} \backslash \bar{\Omega}$, and $\partial \Omega$, respectively (with the convention $\left.H^{0}=L^{2}\right)$. Spaces of vector functions will be denoted by boldface letters; thus $\boldsymbol{H}^{s}=\left(H^{s}\right)^{3}$. Moreover, $\boldsymbol{H}_{\mathrm{t}}^{s}(\partial \Omega):=\left\{\boldsymbol{j} \in \boldsymbol{H}^{s}(\partial \Omega) ; \boldsymbol{j} \cdot \boldsymbol{n}=0\right\}$ denotes the Sobolev space of tangential vector fields of order $s \in \mathbb{R}$. If $\mathrm{D}$ is a differential operator, we write

$$
\begin{aligned}
\boldsymbol{H}(\mathrm{D}, \Omega) & =\left\{\boldsymbol{v} \in \boldsymbol{L}^{2}(\Omega): \mathrm{D} \boldsymbol{v} \in \boldsymbol{L}^{2}(\Omega)\right\} \\
\boldsymbol{H}_{\mathrm{loc}}\left(\mathrm{D}, \mathbb{R}^{3} \backslash \bar{\Omega}\right) & =\left\{\boldsymbol{v} \in \boldsymbol{L}_{\mathrm{loc}}^{2}\left(\mathbb{R}^{3} \backslash \bar{\Omega}\right): \mathrm{D} \boldsymbol{v} \in \boldsymbol{L}_{\mathrm{loc}}^{2}\left(\mathbb{R}^{3} \backslash \bar{\Omega}\right)\right\} .
\end{aligned}
$$

The space $\boldsymbol{H}(\mathrm{D}, \Omega)$ is endowed with the natural graph norm $\|\boldsymbol{v}\|_{\boldsymbol{H}(\mathrm{D}, \Omega)}:=\left(\|\boldsymbol{v}\|_{L^{2}(\Omega)}^{2}+\right.$ $\left.\|\mathrm{D} \boldsymbol{v}\|_{L^{2}(\Omega)}^{2}\right)^{1 / 2}$. This defines in particular the Hilbert spaces $\boldsymbol{H}(\mathbf{c u r l}, \Omega)$ and $\boldsymbol{H}(\mathbf{c u r l}$ curl, $\Omega)$ and the Fréchet spaces $\boldsymbol{H}_{\text {loc }}\left(\operatorname{curl}, \mathbb{R}^{3} \backslash \bar{\Omega}\right)$ and $\boldsymbol{H}_{\text {loc }}\left(\operatorname{curl}\right.$ curl, $\left.\mathbb{R}^{3} \backslash \bar{\Omega}\right)$. Analogously, we introduce the Hilbert space $\boldsymbol{H}_{\mathrm{div}}^{-\frac{1}{2}}(\partial \Omega)=\left\{\boldsymbol{j} \in \boldsymbol{H}_{\mathrm{t}}^{-\frac{1}{2}}(\partial \Omega) ; \operatorname{div}_{\partial \Omega} \boldsymbol{j} \in H^{-\frac{1}{2}}(\partial \Omega)\right\}$ endowed with the norm $\|\boldsymbol{j}\|_{\boldsymbol{H}_{\mathrm{div}}^{-\frac{1}{2}}(\partial \Omega)}=\left(\|\boldsymbol{j}\|_{\boldsymbol{H}^{-\frac{1}{2}(\partial \Omega)}}^{2}+\left\|\operatorname{div}_{\partial \Omega} \boldsymbol{j}\right\|_{H^{-\frac{1}{2}}(\partial \Omega)}^{2}\right)^{1 / 2}$.

\section{Forward and inverse problems.}

2.1. Forward problems. We assume the electromagnetic scatterers are represented by a bounded domain $\Omega$ in $\mathbb{R}^{3}$. Let $\boldsymbol{n}$ denote the outward unit normal vector to the boundary $\partial \Omega$. The domain $\Omega$ consists of $N \geq 1$ disjoint bounded obstacles $\Omega_{j}$ with a smooth closed simply connected boundary $\partial \Omega_{j}$. We have $\partial \Omega=\cup_{j=1}^{N} \partial \Omega_{j}$ and $\partial \Omega_{i} \cap \partial \Omega_{j}=\varnothing$ if $i \neq j$. Let 
$\kappa$ denote the exterior wavenumber. The propagation of electromagnetic waves is governed by the system of Maxwell equations, and the time-harmonic Maxwell system can be reduced to a second order equation for the electric field only [42]. The forward problem is formulated as follows: Given an incident electric wave $\boldsymbol{E}^{\text {inc }}$ which is assumed to solve the second order Maxwell equation in the absence of any scatterer, find the total field $\boldsymbol{E}$ that satisfies either the perfect conductor problem,

(1) $\begin{cases}\operatorname{curl} \operatorname{curl} \boldsymbol{E}-\kappa^{2} \boldsymbol{E}=\mathbf{0} & \text { in } \mathbb{R}^{3} \backslash \bar{\Omega}, \\ \boldsymbol{n} \times \boldsymbol{E}=\mathbf{0} & \text { on } \partial \Omega, \\ \lim _{|\mathbf{x}| \rightarrow+\infty}|\mathbf{x}|\left|\operatorname{curl}\left(\boldsymbol{E}(\mathbf{x})-\boldsymbol{E}^{\mathrm{inc}}(\mathbf{x})\right) \times \frac{\mathbf{x}}{|\mathbf{x}|}-i \kappa\left(\boldsymbol{E}(\mathbf{x})-\boldsymbol{E}^{\mathrm{inc}}(\mathbf{x})\right)\right|=0, & \end{cases}$

or the impedance problem,

$$
\begin{cases}\operatorname{curl} \operatorname{curl} \boldsymbol{E}-\kappa^{2} \boldsymbol{E}=\mathbf{0} & \text { in } \mathbb{R}^{3} \backslash \bar{\Omega} \\ \boldsymbol{n} \times \operatorname{curl} \boldsymbol{E}-i \kappa \lambda \boldsymbol{n} \times \boldsymbol{E} \times \boldsymbol{n}=\mathbf{0} & \text { on } \partial \Omega, \\ \lim _{|\mathbf{x}| \rightarrow+\infty}|\mathbf{x}|\left|\operatorname{curl}\left(\boldsymbol{E}(\mathbf{x})-\boldsymbol{E}^{\mathrm{inc}}(\mathbf{x})\right) \times \frac{\mathbf{x}}{|\mathbf{x}|}-i \kappa\left(\boldsymbol{E}(\mathbf{x})-\boldsymbol{E}^{\text {inc }}(\mathbf{x})\right)\right|=0, & \end{cases}
$$

where $\lambda$ is a complex-valued constant on the surface of each obstacle. The condition $\operatorname{Re}(\lambda)>0$ makes the scatterer absorbing, and the condition $\lambda=0$ refers to the Neumann problem. In the case of dielectric scatterers, we set $\kappa_{e}=\kappa$, and we denote by $\kappa_{i}$ the interior wavenumber and by $\alpha_{e}^{-1}$ and $\alpha_{i}^{-1}$ the exterior and interior magnetic permeabilities, respectively. Setting

$$
\beta_{e}:=\kappa_{e} \sqrt{\alpha_{e}}, \quad \beta_{i}:=\kappa_{i} \sqrt{\alpha_{i}}
$$

the total field satisfies

$$
\begin{cases}\alpha_{e} \operatorname{curl} \operatorname{curl} \boldsymbol{E}-\beta_{e}^{2} \boldsymbol{E}=\mathbf{0} & \text { in } \mathbb{R}^{3} \backslash \bar{\Omega} \\ \alpha_{i} \operatorname{curl} \operatorname{curl} \boldsymbol{E}-\beta_{i}^{2} \boldsymbol{E}=\mathbf{0} & \text { in } \Omega, \\ \boldsymbol{n} \times \boldsymbol{E}^{-}-\boldsymbol{n} \times \boldsymbol{E}^{+}=\mathbf{0} & \text { on } \partial \Omega \\ \alpha_{i} \boldsymbol{n} \times \operatorname{curl} \boldsymbol{E}^{-}-\alpha_{e} \boldsymbol{n} \times \operatorname{curl} \boldsymbol{E}^{+}=\mathbf{0} & \text { on } \partial \Omega \\ \lim _{|\mathbf{x}| \rightarrow+\infty}|\mathbf{x}|\left|\operatorname{curl}\left(\boldsymbol{E}(\mathbf{x})-\boldsymbol{E}^{\mathrm{inc}}(\mathbf{x})\right) \times \frac{\mathbf{x}}{|\mathbf{x}|}-i \kappa_{e}\left(\boldsymbol{E}(\mathbf{x})-\boldsymbol{E}^{\mathrm{inc}}(\mathbf{x})\right)\right|=0, & \end{cases}
$$

where the superscripts "+" and "-" denote limits from the exterior and the interior of $\Omega$, respectively.

The Silver-Müller radiation condition (the last equation in (1), (2), and (3)), which is satisfied uniformly in all directions $\widehat{\mathbf{x}}=\frac{\mathbf{x}}{|\mathbf{x}|}$, implies that the scattered field has an asymptotic behavior of the form (see [14, Theorem 6.8])

$$
\boldsymbol{E}(\mathbf{x})-\boldsymbol{E}^{\mathrm{inc}}(\mathbf{x})=\frac{e^{i \kappa|\mathbf{x}|}}{|\mathbf{x}|} \boldsymbol{E}^{\infty}(\widehat{\mathbf{x}})+O\left(\frac{1}{|\mathbf{x}|}\right), \quad|\mathbf{x}| \rightarrow \infty
$$

uniformly in all directions $\widehat{\mathbf{x}}=\frac{\mathbf{x}}{|\mathbf{x}|}$. The far-field pattern $\boldsymbol{E}^{\infty}$ is a tangential vector function defined on the unit sphere $\mathbb{S}^{2}$ of $\mathbb{R}^{3}$ and is always analytic. 
2.2. The Dirichlet-to-Neumann map. Recall that for $\boldsymbol{u} \in \boldsymbol{H}(\operatorname{curl}, \Omega) \cap \boldsymbol{H}(\operatorname{curl} \operatorname{curl}, \Omega)$ the traces $\boldsymbol{n} \times \boldsymbol{u}_{\mid \partial \Omega}$ and $\boldsymbol{n} \times \operatorname{curl} \boldsymbol{u}_{\mid \partial \Omega}$ are in $\boldsymbol{H}_{\mathrm{div}}^{-1 / 2}(\partial \Omega)$ [7]. These trace results allow us to introduce the Dirichlet-to-Neumann (DtN) operator, which is helpful in reformulating the forward problem in weak form.

Definition 1. Let $B_{R}$ be the ball of radius $R>0$ centered at the origin. We denote by $\boldsymbol{n}_{R}$ the inward unit normal vector to $B_{R}$. For any $\boldsymbol{f} \in \boldsymbol{H}_{\mathrm{div}}^{-1 / 2}\left(\partial B_{R}\right)$ let $\mathbf{U} \in \boldsymbol{H}_{\mathrm{loc}}\left(\mathbf{c u r l}, \mathbb{R}^{3} \backslash \overline{B_{R}}\right) \cap$ $\boldsymbol{H}_{\text {loc }}\left(\operatorname{curl}\right.$ curl, $\left.\mathbb{R}^{3} \backslash \overline{B_{R}}\right)$ satisfy

$$
\begin{cases}\operatorname{curl} \operatorname{curl} \mathbf{U}-\kappa^{2} \mathbf{U}=\mathbf{0} & \text { in } \mathbb{R}^{3} \backslash \overline{B_{R}} \\ \boldsymbol{n}_{R} \times \mathbf{U}=\boldsymbol{f} & \text { on } \partial B_{R} \\ \lim _{|\mathbf{x}| \rightarrow \infty}|\mathbf{x}|\left|\operatorname{curl} \mathbf{U} \times \frac{\mathbf{x}}{|\mathbf{x}|}-i \kappa \mathbf{U}\right|=0 . & \end{cases}
$$

Then the DtN map is defined by

$$
\begin{aligned}
L_{\kappa}: \quad \boldsymbol{H}_{\mathrm{div}}^{-1 / 2}\left(\partial B_{R}\right) & \rightarrow \boldsymbol{H}_{\mathrm{div}}^{-1 / 2}\left(\partial B_{R}\right), \\
\boldsymbol{f}=\boldsymbol{n} \times \mathbf{U} & \mapsto \frac{1}{i \kappa} \boldsymbol{n} \times \operatorname{curl} \mathbf{U} .
\end{aligned}
$$

Notice that the DtN map satisfies, thanks to second Green's formula,

$$
\int_{\partial B_{R}} L_{\kappa}\left(\boldsymbol{n}_{R} \times \mathbf{U}_{1}\right) \cdot \mathbf{U}_{2}=\int_{\partial B_{R}} L_{\kappa}\left(\boldsymbol{n}_{R} \times \mathbf{U}_{2}\right) \cdot \mathbf{U}_{1} .
$$

We obtain the following correspondence result [41, Chapter 10].

Proposition 2. Let $R>0$ be such that $\bar{\Omega} \subset B_{R}$.

(i) Dirichlet problem: We denote by $\widetilde{\boldsymbol{E}} \in \boldsymbol{H}\left(\mathbf{c u r l}, B_{R} \backslash \bar{\Omega}\right) \cap \boldsymbol{H}\left(\mathbf{c u r l} \mathbf{c u r l}, B_{R} \backslash \bar{\Omega}\right)$ the solution to

$$
\begin{cases}\operatorname{curl} \operatorname{curl} \widetilde{\boldsymbol{E}}-\kappa^{2} \widetilde{\boldsymbol{E}}=\mathbf{0} & \text { in } B_{R} \backslash \bar{\Omega}, \\ \boldsymbol{n} \times \widetilde{\boldsymbol{E}}=\mathbf{0} & \text { on } \partial \Omega, \\ \boldsymbol{n}_{R} \times \operatorname{curl}\left(\widetilde{\boldsymbol{E}}-\boldsymbol{E}^{\mathrm{inc}}\right)=i \kappa L_{\kappa}\left(\boldsymbol{n}_{R} \times\left(\widetilde{\boldsymbol{E}}-\boldsymbol{E}^{\mathrm{inc}}\right)\right) & \text { on } \partial B_{R} .\end{cases}
$$

Then we have $\widetilde{\boldsymbol{E}}=\boldsymbol{E}_{\mid B_{R} \backslash \bar{\Omega}}$, where $\boldsymbol{E}_{\left|B_{R}\right| \bar{\Omega}}$ is the restriction to $B_{R} \backslash \bar{\Omega}$ of the solution to the forward problem (1). Reciprocally, the solution to (6) can be extended to $\mathbb{R}^{3} \backslash \bar{B}_{R}$ using the DtN map to recover the solution of problem (1).

(ii) Impedance problem: We denote by $\widetilde{\boldsymbol{E}} \in \boldsymbol{H}\left(\mathbf{c u r l}, B_{R} \backslash \bar{\Omega}\right) \cap \boldsymbol{H}\left(\mathbf{c u r l} \mathbf{c u r l}, B_{R} \backslash \bar{\Omega}\right)$ the solution to

$$
\begin{cases}\operatorname{curl} \operatorname{curl} \widetilde{\boldsymbol{E}}-\kappa^{2} \widetilde{\boldsymbol{E}}=\mathbf{0} & \text { in } B_{R} \backslash \bar{\Omega}, \\ \boldsymbol{n} \times \operatorname{curl} \widetilde{\boldsymbol{E}}-i \kappa \lambda \boldsymbol{n} \times \widetilde{\boldsymbol{E}} \times \boldsymbol{n}=\mathbf{0} & \text { on } \partial \Omega, \\ \boldsymbol{n}_{R} \times \operatorname{curl}\left(\widetilde{\boldsymbol{E}}-\boldsymbol{E}^{\text {inc }}\right)=i \kappa L_{\kappa}\left(\boldsymbol{n}_{R} \times\left(\widetilde{\boldsymbol{E}}-\boldsymbol{E}^{\text {inc }}\right)\right) & \text { on } \partial B_{R} .\end{cases}
$$

Then we have $\widetilde{\boldsymbol{E}}=\boldsymbol{E}_{\mid B_{R} \backslash \bar{\Omega}}$, where $\boldsymbol{E}_{\mid B_{R} \backslash \bar{\Omega}}$ is the restriction to $B_{R} \backslash \bar{\Omega}$ of the solution to the forward problem (2). Reciprocally, the solution to (7) can be extended to $\mathbb{R}^{3} \backslash \bar{B}_{R}$ using the DtN map to recover the solution of problem (2). Recall that the Neumann boundary condition corresponds to the particular case $\lambda=0$. 
(iii) Transmission problem: We denote by $\widetilde{\boldsymbol{E}} \in \boldsymbol{H}\left(\mathbf{c u r l}, B_{R}\right) \cap \boldsymbol{H}\left(\mathbf{c u r l}\right.$ curl, $\left.B_{R}\right)$ the solution to

$$
\begin{cases}\alpha_{e} \operatorname{curl} \operatorname{curl} \widetilde{\boldsymbol{E}}-\beta_{e}^{2} \widetilde{\boldsymbol{E}}=\mathbf{0} & \text { in } B_{R} \backslash \bar{\Omega}, \\ \alpha_{i} \operatorname{curl} \operatorname{curl} \widetilde{\boldsymbol{E}}-\beta_{i}^{2} \widetilde{\boldsymbol{E}}=\mathbf{0} & \text { in } \Omega, \\ \boldsymbol{n} \times \widetilde{\boldsymbol{E}}^{-}-\boldsymbol{n} \times \widetilde{\boldsymbol{E}}^{+}=\mathbf{0} & \text { on } \partial \Omega, \\ \alpha_{i} \boldsymbol{n} \times \operatorname{curl} \widetilde{\boldsymbol{E}}^{-}-\alpha_{e} \boldsymbol{n} \times \operatorname{curl} \widetilde{\boldsymbol{E}}^{+}=\mathbf{0} & \text { on } \partial \Omega, \\ \boldsymbol{n}_{R} \times \operatorname{curl}\left(\widetilde{\boldsymbol{E}}-\boldsymbol{E}^{\mathrm{inc}}\right)=i \kappa_{e} L_{\kappa_{e}}\left(\boldsymbol{n}_{R} \times\left(\widetilde{\boldsymbol{E}}-\boldsymbol{E}^{\mathrm{inc}}\right)\right) & \text { on } \partial B_{R} .\end{cases}
$$

Then we have $\widetilde{\boldsymbol{E}}=\boldsymbol{E}_{\mid B_{R}}$, where $\boldsymbol{E}_{\mid B_{R}}$ is the restriction to $B_{R}$ of the solution to the forward problem (3). Reciprocally, the solution to (8) can be extended to $\mathbb{R}^{3} \backslash \bar{B}_{R}$ using the DtN map to recover the solution of problem (3).

2.3. Inverse problems. In this paper we measure near-field or far-field data from the scattering of $N_{\text {inc }}$ incident waves, as, for example, plane waves of the form $\boldsymbol{E}_{r}^{\mathrm{inc}}(\mathbf{x})=\boldsymbol{p}_{r} e^{i \kappa \mathbf{x} \cdot \boldsymbol{d}_{r}}$, $r=1, \ldots, N_{\text {inc }}$, where $\boldsymbol{d}_{r}, \boldsymbol{p}_{r} \in \mathbb{S}^{2}$ and $\boldsymbol{d}_{r} \cdot \boldsymbol{p}_{r}=0$. We denote by $\boldsymbol{E}_{r}$ the total field solution to one of the problems (1), (2), or (3) for the incident wave $\boldsymbol{E}_{r}^{\text {inc }}$, and by $\boldsymbol{E}_{\text {meas, } r}$ the measured total field. The inverse problem consists in recovering the location and the approximated shapes of the scatterers through the minimization of the nonlinear cost function,

$$
J\left(\mathbb{R}^{3} \backslash \Omega\right)=\sum_{r=1}^{N_{\text {inc }}} \frac{1}{2} \int_{\Gamma_{\text {meas }}}\left|\boldsymbol{E}_{r}\left(\mathbf{y}_{\text {obs }}\right)-\boldsymbol{E}_{\text {meas }, r}\left(\mathbf{y}_{\text {obs }}\right)\right|^{2} d \mu\left(\mathbf{y}_{\text {obs }}\right),
$$

using the topological gradient method. The measure $\mu$ may represent the Lebesgue measure on a surface $\Gamma_{\text {meas }}$ of $\mathbb{R}^{3}$ or, in a more realistic context, it may represent a finite sum of Dirac measures. In the later case, the cost function reads

$$
J\left(\mathbb{R}^{3} \backslash \Omega\right)=\sum_{r=1}^{N_{\text {inc }}} \frac{1}{2} \sum_{s=1}^{N_{\text {obs }}}\left|\boldsymbol{E}_{r}\left(\mathbf{y}_{\text {obs }}^{s}\right)-\boldsymbol{E}_{\text {meas }, r}\left(\mathbf{y}_{\text {obs }}^{s}\right)\right|^{2},
$$

where $\mathbf{y}_{\text {obs }}^{s}$, for $s=1, \ldots, N_{\text {obs }}$, are a finite number of observation points in $\mathbb{R}^{3}$. If we use far-field data, then the cost function is

$$
J\left(\mathbb{R}^{3} \backslash \Omega\right)=\sum_{r=1}^{N_{\text {inc }}} \frac{1}{2} \sum_{s=1}^{N_{\text {obs }}}\left|\boldsymbol{E}_{r}^{\infty}\left(\widehat{\mathbf{y}}_{\text {obs }}^{s}\right)-\boldsymbol{E}_{\text {meas }, r}^{\infty}\left(\widehat{\mathbf{y}}_{\text {obs }}^{s}\right)\right|^{2},
$$

where $\widehat{\mathbf{y}}_{\text {obs }}^{s}$, for $s=1, \ldots, N_{\text {obs }}$, are a finite number of observation points in $\mathbb{S}^{2}$.

3. Topological derivative of a constrained shape functional. The topological derivative (TD) of a constrained shape function $\mathcal{J}(\mathcal{R})=\mathcal{J}\left(u_{\mathcal{R}}\right)$, where $\mathcal{R}$ is a domain of $\mathbb{R}^{3}$ and $u_{\mathcal{R}}$ is the solution to a given partial differential equation problem defined in $\mathcal{R}$, is a scalar field $\mathcal{D}_{T}$ that measures the sensitivity of the functional when an infinitesimal ball is located at each point $\mathbf{x} \in \mathcal{R}$ [51]. It provides at each point $\mathbf{x} \in \mathcal{R}$ an asymptotic expansion of the form

$$
\mathcal{J}\left(\mathcal{R} \backslash B_{\varepsilon}(\mathbf{x})\right)=\mathcal{J}(\mathcal{R})+f(\varepsilon) \mathcal{D}_{T}(\mathbf{x})+o(f(\varepsilon)), \quad \varepsilon \rightarrow 0,
$$


where $f>0$ is a monotonically decreasing function such that $f(\varepsilon) \rightarrow 0$ as $\varepsilon \rightarrow 0$. As we will shortly see, for the Maxwell problems (with Dirichlet, Neumann, impedance, and transmission conditions) one can take $f$ as the volume of the ball, $f(\varepsilon)=\frac{4}{3} \pi \varepsilon^{3}$. This expansion motivates the reconstruction technique: if we place objects in the regions where the TD takes pronounced negative values, the error functional is expected to decrease, yielding a prediction of the number, location, size, and even shape of the scatterers.

To derive closed formulae for the TD, we adopt the methodology proposed in [44], where the TD is obtained from classical shape sensitivity computations, i.e., as a limit of shape derivatives. Direct computations in terms of Green functions are performed in [24, 25], while asymptotic expansions are exploited in $[40,52,53]$.

As proved in [44], the TD can be found as a limit of shape derivatives. The procedure is the following. Given a point $\mathbf{x} \in \mathcal{R}$ and $\varepsilon>0$ small enough to guarantee that $\overline{B_{\varepsilon}(\mathbf{x})} \subset \mathcal{R}$, we define a vector field $\mathbf{V}$ such that

$$
\mathbf{V}(\mathbf{z})=\boldsymbol{n}_{\varepsilon}(\mathbf{z}) \quad \forall \mathbf{z} \in \partial B_{\varepsilon}(\mathbf{x}),
$$

$\boldsymbol{n}_{\varepsilon}$ being the outward normal vector at the boundary of the ball, and such that it vanishes outside a narrow neighborhood of $\partial B_{\varepsilon}(\mathbf{x})$ (in particular, $\mathbf{V}=\mathbf{0}$ on $\partial \mathcal{R}$ ). Now we consider a family of deformations conducted by a parameter $\tau>0$,

$$
\varphi_{\tau}(\mathbf{z}):=\mathbf{z}+\tau \mathbf{V}(\mathbf{z}), \quad \mathbf{z} \in \mathbb{R}^{3} .
$$

Then, the TD of the functional $\mathcal{J}(\mathcal{R})$ at the point $\mathrm{x} \in \mathcal{R}$ is given by

$$
\mathcal{D}_{T}(\mathbf{x})=\left.\lim _{\varepsilon \rightarrow 0} \frac{1}{f^{\prime}(\varepsilon)} \frac{d}{d \tau} \mathcal{J}\left(\varphi_{\tau}\left(\mathcal{R} \backslash B_{\varepsilon}(\mathbf{x})\right)\right)\right|_{\tau=0},
$$

where $f^{\prime}$ is the derivative of the function $f$ appearing in the definition of the TD (11).

For the shape functionals of the form (9) considered in this paper it is clear that by linearity we can assume without loss of generality that $N_{\text {inc }}=1$ (only one incident wave is generated); we consider the functional

$$
J\left(\mathbb{R}^{3} \backslash \Omega\right)=\frac{1}{2} \int_{\Gamma_{\text {meas }}}\left|\boldsymbol{E}-\boldsymbol{E}_{\text {meas }}\right|^{2} d \mu
$$

where $\boldsymbol{E}$ solves one of the problems (1), (2), or (3) for a given incident wave.

In the absence of an initial guess for $\Omega$, we study the functional over $\mathbb{R}^{3}$. Notice that, defining

$$
\Omega_{\varepsilon, \tau}:=\varphi_{\tau}\left(B_{\varepsilon}(\mathbf{x})\right)
$$

it follows that

$$
\begin{array}{cl}
\varphi_{\tau}\left(\mathbb{R}^{3} \backslash B_{\varepsilon}(\mathbf{x})\right)=\mathbb{R}^{3} \backslash \Omega_{\varepsilon, \tau}, & \varphi_{\tau}\left(B_{R} \backslash B_{\varepsilon}(\mathbf{x})\right)=B_{R} \backslash \Omega_{\varepsilon, \tau}, \\
\varphi_{\tau}\left(\partial B_{R}\right)=\partial B_{R}, & \varphi_{\tau}\left(\Gamma_{\text {meas }}\right)=\Gamma_{\text {meas }} .
\end{array}
$$

The cost functional in the transformed domain is

$$
J\left(\varphi_{\tau}\left(\mathbb{R}^{3} \backslash B_{\varepsilon}(\mathbf{x})\right)\right)=J\left(\mathbb{R}^{3} \backslash \Omega_{\varepsilon, \tau}\right)=\frac{1}{2} \int_{\Gamma_{\text {meas }}}\left|\boldsymbol{E}_{\varepsilon, \tau}-\boldsymbol{E}_{\text {meas }}\right|^{2} d \mu,
$$


with $\boldsymbol{E}_{\varepsilon, \tau}$ solving (1), (2), or (3) (depending on the considered boundary condition) with $\Omega=\Omega_{\varepsilon, \tau}$. When $\tau=0$, the deformation $\varphi_{0}$ is the identity, $\Omega_{\varepsilon}:=\Omega_{\varepsilon, 0}=B_{\varepsilon}(\mathbf{x})$, and $\boldsymbol{E}_{\varepsilon}:=\boldsymbol{E}_{\varepsilon, 0}$ solves the forward problem (1), (2), or (3) with $\Omega=B_{\varepsilon}(\mathbf{x})$. In any of the three cases,

$$
\left.\frac{d}{d \tau} J\left(\mathbb{R}^{3} \backslash \Omega_{\varepsilon, \tau}\right)\right|_{\tau=0}=\operatorname{Re}\left[\int_{\Gamma_{\text {meas }}} \overline{\left(\boldsymbol{E}_{\varepsilon}-\boldsymbol{E}_{\text {meas }}\right)} \cdot \dot{\boldsymbol{E}}_{\varepsilon} d \mu\right]
$$

where $\dot{\boldsymbol{E}}_{\varepsilon}=\left.\frac{d}{d \tau} \boldsymbol{E}_{\varepsilon, \tau}\right|_{\tau=0}$ (see [28]). In the next theorems we give equivalent expressions of the shape derivative (13) for the different boundary conditions considered in this paper.

Theorem 3. For the Dirichlet problem,

$$
\left.\frac{d}{d \tau} J\left(\mathbb{R}^{3} \backslash \Omega_{\varepsilon, \tau}\right)\right|_{\tau=0}=\operatorname{Re}\left[\int_{\partial B_{\varepsilon}(\mathbf{x})}\left(\boldsymbol{n}_{\varepsilon} \times \operatorname{curl} \boldsymbol{E}_{\varepsilon}\right) \cdot\left(\boldsymbol{n}_{\varepsilon} \times \operatorname{curl} \overline{\boldsymbol{W}}_{\varepsilon}\right)-\kappa^{2}\left(\boldsymbol{n}_{\varepsilon} \cdot \boldsymbol{E}_{\varepsilon}\right)\left(\boldsymbol{n}_{\varepsilon} \cdot \overline{\boldsymbol{W}}_{\varepsilon}\right)\right],
$$

where $\boldsymbol{E}_{\varepsilon}$ solves the forward problem (1) with $\Omega=B_{\varepsilon}(\mathbf{x})$ and $\overline{\boldsymbol{W}}_{\varepsilon}$ is the complex-conjugate of the solution $\boldsymbol{W}_{\varepsilon}$ of the associated adjoint problem; that is, it solves

$$
\begin{cases}\operatorname{curl} \operatorname{curl} \overline{\boldsymbol{W}}_{\varepsilon}-\kappa^{2} \overline{\boldsymbol{W}}_{\varepsilon}=\left(\overline{\boldsymbol{E}_{\text {meas }}-\boldsymbol{E}_{\varepsilon}}\right) \delta_{\Gamma_{\text {meas }}} & \text { in } \mathbb{R}^{3} \backslash \overline{B_{\varepsilon}(\mathbf{x}),} \\ \boldsymbol{n}_{\varepsilon} \times \overline{\boldsymbol{W}}_{\varepsilon}=\mathbf{0} & \text { on } \partial B_{\varepsilon}(\mathbf{x}), \\ \lim _{|\mathbf{z}| \rightarrow \infty}|\mathbf{z}|\left|\operatorname{curl} \overline{\boldsymbol{W}}_{\varepsilon}(\mathbf{z}) \times \frac{\mathbf{z}}{|\mathbf{z}|}-i \kappa \overline{\boldsymbol{W}}_{\varepsilon}(\mathbf{z})\right|=0 . & \end{cases}
$$

Proof. We use that $\dot{\boldsymbol{E}}_{\varepsilon}$ solves [33, Theorem 10.1]:

$$
\begin{cases}\operatorname{curl} \operatorname{curl} \dot{\boldsymbol{E}}_{\varepsilon}-\kappa^{2} \dot{\boldsymbol{E}}_{\varepsilon}=\mathbf{0} & \text { in } \mathbb{R}^{3} \backslash \overline{B_{\varepsilon}(\mathbf{x})}, \\ \boldsymbol{n}_{\varepsilon} \times \dot{\boldsymbol{E}}_{\varepsilon}=-\boldsymbol{n}_{\varepsilon} \times\left(\operatorname{curl} \boldsymbol{E}_{\varepsilon} \times \boldsymbol{n}_{\varepsilon}\right)-\boldsymbol{n}_{\varepsilon} \times \boldsymbol{\nabla}\left(\boldsymbol{n}_{\varepsilon} \cdot \boldsymbol{E}_{\varepsilon}\right) & \text { on } \partial B_{\varepsilon}(\mathbf{x}), \\ \lim _{|\mathbf{z}| \rightarrow \infty}|\mathbf{z}|\left|\operatorname{curl} \dot{\boldsymbol{E}}_{\varepsilon}(\mathbf{z}) \times \frac{\mathbf{z}}{|\mathbf{z}|}-i \kappa \dot{\boldsymbol{E}}_{\varepsilon}(\mathbf{z})\right|=0 & \end{cases}
$$

We take now $R>0$ such that $\overline{B_{\varepsilon}(\mathbf{x})} \subset B_{R}$. By Green's formula in $B_{R} \backslash \overline{B_{\varepsilon}(\mathbf{x})}$ [7, section 3.2],

(16) $\int_{B_{R} \backslash \overline{B_{\varepsilon}(\mathbf{x})}} \operatorname{curl} \mathbf{U}_{1} \cdot \operatorname{curl} \overline{\mathbf{U}}_{2}=\int_{B_{R} \backslash \overline{B_{\varepsilon}(\mathbf{x})}} \operatorname{curl} \operatorname{curl} \mathbf{U}_{1} \cdot \overline{\mathbf{U}}_{2}+\int_{\partial B_{R} \cup \partial B_{\varepsilon}(\mathbf{x})} \boldsymbol{\nu} \times \operatorname{curl} \mathbf{U}_{1} \cdot \overline{\mathbf{U}}_{2}$

for all $\mathbf{U}_{1}, \mathbf{U}_{2} \in \boldsymbol{H}\left(\mathbf{c u r l}, B_{R} \backslash \bar{\Omega}\right) \cap \boldsymbol{H}\left(\mathbf{c u r l} \mathbf{c u r l}, B_{R} \backslash \bar{\Omega}\right)$, where $\boldsymbol{\nu}$ is the inward unit normal vector to $B_{R} \backslash \overline{B_{\varepsilon}(\mathbf{x})}$. Then, taking into account that the DtN map satisfies the identity (5), it follows that

$$
\begin{aligned}
& \int_{B_{R} \backslash \overline{B_{\varepsilon}(\mathbf{x})}} \operatorname{curl} \operatorname{curl} \dot{\boldsymbol{E}}_{\varepsilon} \cdot \overline{\boldsymbol{W}}_{\varepsilon}-\kappa^{2} \dot{\boldsymbol{E}}_{\varepsilon} \cdot \overline{\boldsymbol{W}}_{\varepsilon}+\int_{\partial B_{\varepsilon}(\mathbf{x})}\left(\boldsymbol{n}_{\varepsilon} \times \operatorname{curl} \dot{\boldsymbol{E}}_{\varepsilon}\right) \cdot \overline{\boldsymbol{W}}_{\varepsilon} \\
& \quad=\int_{B_{R} \backslash \overline{B_{\varepsilon}(\mathbf{x})}} \operatorname{curl} \operatorname{curl} \overline{\boldsymbol{W}}_{\varepsilon} \cdot \dot{\boldsymbol{E}}_{\varepsilon}-\kappa^{2} \overline{\boldsymbol{W}}_{\varepsilon} \cdot \dot{\boldsymbol{E}}_{\varepsilon}+\int_{\partial B_{\varepsilon}(\mathbf{x})}\left(\boldsymbol{n}_{\varepsilon} \times \operatorname{curl} \overline{\boldsymbol{W}}_{\varepsilon}\right) \cdot \dot{\boldsymbol{E}}_{\varepsilon} .
\end{aligned}
$$


The left-hand side in (17) is equal to zero. Then,

$$
\begin{aligned}
& \int_{\Gamma_{\text {meas }}}\left(\overline{\boldsymbol{E}_{\varepsilon}-\boldsymbol{E}_{\text {meas }}}\right) \cdot \dot{\boldsymbol{E}}_{\varepsilon} d \mu=\int_{\partial B_{\varepsilon}(\mathbf{x})}\left(\boldsymbol{n}_{\varepsilon} \times \operatorname{curl} \overline{\boldsymbol{W}}_{\varepsilon}\right) \cdot \dot{\boldsymbol{E}}_{\varepsilon} \\
& =-\int_{\partial B_{\varepsilon}(\mathbf{x})} \operatorname{curl} \overline{\boldsymbol{W}}_{\varepsilon} \cdot\left(\boldsymbol{n}_{\varepsilon} \times \dot{\boldsymbol{E}}_{\varepsilon}\right) \\
& =\int_{\partial B_{\varepsilon}(\mathbf{x})} \operatorname{curl} \overline{\boldsymbol{W}}_{\varepsilon} \cdot\left(\boldsymbol{n}_{\varepsilon} \times\left(\operatorname{curl} \boldsymbol{E}_{\varepsilon} \times \boldsymbol{n}_{\varepsilon}\right)+\boldsymbol{n}_{\varepsilon} \times \nabla\left(\boldsymbol{n}_{\varepsilon} \cdot \boldsymbol{E}_{\varepsilon}\right)\right) \\
& =\int_{\partial B_{\varepsilon}(\mathbf{x})}\left(\boldsymbol{n}_{\varepsilon} \times \operatorname{curl} \boldsymbol{E}_{\varepsilon}\right) \cdot\left(\boldsymbol{n}_{\varepsilon} \times \operatorname{curl} \overline{\boldsymbol{W}}_{\varepsilon}\right)-\kappa^{2}\left(\boldsymbol{n}_{\varepsilon} \cdot \boldsymbol{E}_{\varepsilon}\right)\left(\boldsymbol{n}_{\varepsilon} \cdot \overline{\boldsymbol{W}}_{\varepsilon}\right),
\end{aligned}
$$

using $\boldsymbol{n}_{\varepsilon} \cdot \operatorname{curl} \operatorname{curl} \overline{\boldsymbol{W}}_{\varepsilon}=\kappa^{2} \boldsymbol{n}_{\varepsilon} \cdot \overline{\boldsymbol{W}}_{\varepsilon}$. The result follows then by (13).

Theorem 4. For the impedance problem,

$$
\begin{aligned}
\left.\frac{d}{d \tau} J\left(\mathbb{R}^{3} \backslash \Omega_{\varepsilon, \tau}\right)\right|_{\tau=0}=\operatorname{Re} & {\left[\int_{\partial B_{\varepsilon}(\mathbf{x})} \kappa^{2}\left(1-\lambda^{2}\right)\left(\boldsymbol{n}_{\varepsilon} \times \boldsymbol{E}_{\varepsilon}\right) \cdot\left(\boldsymbol{n}_{\varepsilon} \times \overline{\boldsymbol{W}}_{\varepsilon}\right)\right.} \\
& \left.+\int_{\partial B_{\varepsilon}(\mathbf{x})}-\left(\boldsymbol{n}_{\varepsilon} \cdot \operatorname{curl} \boldsymbol{E}_{\varepsilon}\right)\left(\boldsymbol{n}_{\varepsilon} \cdot \operatorname{curl} \overline{\boldsymbol{W}}_{\varepsilon}\right)-\kappa^{2}\left(\boldsymbol{n}_{\varepsilon} \cdot \boldsymbol{E}_{\varepsilon}\right)\left(\boldsymbol{n}_{\varepsilon} \cdot \overline{\boldsymbol{W}}_{\varepsilon}\right)\right],
\end{aligned}
$$

where $\boldsymbol{E}_{\varepsilon}$ solves the forward problem (2) with $\Omega=B_{\varepsilon}(\mathbf{x})$ and $\overline{\boldsymbol{W}}_{\varepsilon}$ is the complex-conjugate of the solution $\boldsymbol{W}_{\varepsilon}$ of the associated adjoint problem; that is, it solves

$$
\begin{cases}\operatorname{curl} \operatorname{curl} \overline{\boldsymbol{W}}_{\varepsilon}-\kappa^{2} \overline{\boldsymbol{W}}_{\varepsilon}=\left(\overline{\boldsymbol{E}_{\text {meas }}-\boldsymbol{E}_{\varepsilon}}\right) \delta_{\Gamma_{\text {meas }}} & \text { in } \mathbb{R}^{3} \backslash \overline{B_{\varepsilon}(\mathbf{x})}, \\ \boldsymbol{n}_{\varepsilon} \times \operatorname{curl} \overline{\boldsymbol{W}}_{\varepsilon}-i \kappa \lambda \boldsymbol{n}_{\varepsilon} \times \overline{\boldsymbol{W}}_{\varepsilon} \times \boldsymbol{n}_{\varepsilon}=\mathbf{0} & \text { on } \partial B_{\varepsilon}(\mathbf{x}), \\ \lim _{|\mathbf{z}| \rightarrow \infty}|\mathbf{z}|\left|\operatorname{curl} \overline{\boldsymbol{W}}_{\varepsilon}(\mathbf{z}) \times \frac{\mathbf{z}}{|\mathbf{z}|}-i \kappa \overline{\boldsymbol{W}}_{\varepsilon}(\mathbf{z})\right|=0 . & \end{cases}
$$

For the Neumann problem $(\lambda=0)$, formula (18) reduces to

$$
\left.\frac{d}{d \tau} J\left(\mathbb{R}^{3} \backslash \Omega_{\varepsilon, \tau}\right)\right|_{\tau=0}=\operatorname{Re}\left[\int_{\partial B_{\varepsilon}(\mathbf{x})} \kappa^{2}\left(\boldsymbol{n}_{\varepsilon} \times \boldsymbol{E}_{\varepsilon}\right) \cdot\left(\boldsymbol{n}_{\varepsilon} \times \overline{\boldsymbol{W}}_{\varepsilon}\right)-\left(\boldsymbol{n}_{\varepsilon} \cdot \operatorname{curl} \boldsymbol{E}_{\varepsilon}\right)\left(\boldsymbol{n}_{\varepsilon} \cdot \operatorname{curl} \overline{\boldsymbol{W}}_{\varepsilon}\right)\right] .
$$

Proof. It can be proven that $\dot{\boldsymbol{E}}_{\varepsilon}$ solves (taking $\mathbf{H}=\frac{1}{i \kappa} \operatorname{curl} \boldsymbol{E}_{\varepsilon}$ and $\nu \cdot h=1$ in [26, Theorem 3.4]):

$$
\left\{\begin{array}{cc}
\operatorname{curl} \operatorname{curl} \dot{\boldsymbol{E}}_{\varepsilon}-\kappa^{2} \dot{\boldsymbol{E}}_{\varepsilon}=\mathbf{0} & \text { in } \mathbb{R}^{3} \backslash \overline{B_{\varepsilon}(\mathbf{x})}, \\
\boldsymbol{n}_{\varepsilon} \times \operatorname{curl} \dot{\boldsymbol{E}}_{\varepsilon}-i \kappa \lambda \boldsymbol{n}_{\varepsilon} \times \dot{\boldsymbol{E}}_{\varepsilon} \times \boldsymbol{n}_{\varepsilon} & \\
=-\kappa^{2} \boldsymbol{n}_{\varepsilon} \times\left(\boldsymbol{E}_{\varepsilon} \times \boldsymbol{n}_{\varepsilon}\right)-\boldsymbol{n}_{\varepsilon} \times \boldsymbol{\nabla}\left(\boldsymbol{n}_{\varepsilon} \cdot \operatorname{curl} \boldsymbol{E}_{\varepsilon}\right) & \\
-i \kappa \lambda\left(\boldsymbol{n}_{\varepsilon} \times \operatorname{curl} \boldsymbol{E}_{\varepsilon}-\boldsymbol{\nabla}_{\partial B_{\varepsilon}(\mathbf{x})}\left(\boldsymbol{n}_{\varepsilon} \cdot \boldsymbol{E}_{\varepsilon}\right)\right) & \text { on } \partial B_{\varepsilon}(\mathbf{x}), \\
\lim _{|\mathbf{z}| \rightarrow \infty}|\mathbf{z}|\left|\operatorname{curl} \dot{\boldsymbol{E}}_{\varepsilon}(\mathbf{z}) \times \frac{\mathbf{z}}{|\mathbf{z}|}-i \kappa \dot{\boldsymbol{E}}_{\varepsilon}(\mathbf{z})\right|=0 . &
\end{array}\right.
$$


Applying Green's formula (16) and the identity (5) when $\mathbf{U}_{1}=\dot{\boldsymbol{E}}_{\varepsilon}$ and $\mathbf{U}_{2}=\overline{\boldsymbol{W}}_{\varepsilon}$, we find that (17) holds. Notice now that the first integral on the left-hand side of (17) vanishes. We also have that

$$
\int_{\partial B_{\varepsilon}(\mathbf{x})}\left(\boldsymbol{n}_{\varepsilon} \times \operatorname{curl} \overline{\boldsymbol{W}}_{\varepsilon}\right) \cdot \dot{\boldsymbol{E}}_{\varepsilon}=i \kappa \lambda \int_{\partial B_{\varepsilon}(\mathbf{x})} \overline{\boldsymbol{W}}_{\varepsilon} \cdot\left(\boldsymbol{n}_{\varepsilon} \times \dot{\boldsymbol{E}}_{\varepsilon} \times \boldsymbol{n}_{\varepsilon}\right) .
$$

Hence, using the boundary condition satisfied by $\dot{\boldsymbol{E}}_{\varepsilon}$, we find that (17) can be written in the form

$$
\begin{aligned}
\int_{\Gamma_{\text {meas }}}\left(\overline{\boldsymbol{E}_{\varepsilon}-\boldsymbol{E}_{\text {meas }}}\right) \cdot \dot{\boldsymbol{E}}_{\varepsilon} d \mu= & \int_{\partial B_{\varepsilon}(\mathbf{x})}\left(\kappa^{2} \boldsymbol{n}_{\varepsilon} \times\left(\boldsymbol{E}_{\varepsilon} \times \boldsymbol{n}_{\varepsilon}\right)+\boldsymbol{n}_{\varepsilon} \times \nabla\left(\boldsymbol{n}_{\varepsilon} \cdot \operatorname{curl} \boldsymbol{E}_{\varepsilon}\right)\right) \cdot \overline{\boldsymbol{W}}_{\varepsilon} \\
& +i \kappa \lambda \int_{\partial B_{\varepsilon}(\mathbf{x})}\left(\boldsymbol{n}_{\varepsilon} \times \operatorname{curl} \boldsymbol{E}_{\varepsilon}-\nabla_{\partial B_{\varepsilon}(\mathbf{x})}\left(\boldsymbol{n}_{\varepsilon} \cdot \boldsymbol{E}_{\varepsilon}\right)\right) \cdot \overline{\boldsymbol{W}}_{\varepsilon}
\end{aligned}
$$

We have

$$
\begin{aligned}
i \kappa \lambda \int_{\partial B_{\varepsilon}(\mathbf{x})} \boldsymbol{n}_{\varepsilon} \times \operatorname{curl} \boldsymbol{E}_{\varepsilon} \cdot \overline{\boldsymbol{W}}_{\varepsilon} & =i \kappa \lambda \int_{\partial B_{\varepsilon}(\mathbf{x})} i \kappa \lambda\left(\boldsymbol{n}_{\varepsilon} \times \boldsymbol{E}_{\varepsilon} \times \boldsymbol{n}_{\varepsilon}\right) \cdot \overline{\boldsymbol{W}}_{\varepsilon} \\
& =-\kappa^{2} \lambda^{2} \int_{\partial B_{\varepsilon}(\mathbf{x})}\left(\boldsymbol{n}_{\varepsilon} \times \boldsymbol{E}_{\varepsilon}\right) \cdot\left(\boldsymbol{n}_{\varepsilon} \times \overline{\boldsymbol{W}}_{\varepsilon}\right)
\end{aligned}
$$

and

$$
\begin{aligned}
& -i \kappa \lambda \int_{\partial B_{\varepsilon}(\mathbf{x})} \boldsymbol{\nabla}_{\partial B_{\varepsilon}(\mathbf{x})}\left(\boldsymbol{n}_{\varepsilon} \cdot \boldsymbol{E}_{\varepsilon}\right) \cdot \overline{\boldsymbol{W}}_{\varepsilon}=-i \kappa \lambda \int_{\partial B_{\varepsilon}(\mathbf{x})} \underset{\partial B_{\varepsilon}(\mathbf{x})}{ }\left(\boldsymbol{n}_{\varepsilon} \cdot \boldsymbol{E}_{\varepsilon}\right) \cdot\left(\boldsymbol{n}_{\varepsilon} \times \operatorname{curl} \overline{\boldsymbol{W}}_{\varepsilon} \times \boldsymbol{n}_{\varepsilon}\right) \\
& =-\int_{\partial B_{\varepsilon}(\mathbf{x})} \nabla_{\partial B_{\varepsilon}(\mathbf{x})}\left(\boldsymbol{n}_{\varepsilon} \cdot \boldsymbol{E}_{\varepsilon}\right) \cdot\left(\boldsymbol{n}_{\varepsilon} \times \operatorname{curl} \overline{\boldsymbol{W}}_{\varepsilon}\right) \\
& =\int_{\partial B_{\varepsilon}(\mathbf{x})}\left(\boldsymbol{n}_{\varepsilon} \cdot \boldsymbol{E}_{\varepsilon}\right) \cdot \operatorname{div}_{\partial B_{\varepsilon}(\mathbf{x})}\left(\boldsymbol{n}_{\varepsilon} \times \operatorname{curl} \overline{\boldsymbol{W}}_{\varepsilon}\right) \\
& =-\int_{\partial B_{\varepsilon}(\mathbf{x})}\left(\boldsymbol{n}_{\varepsilon} \cdot \boldsymbol{E}_{\varepsilon}\right) \cdot\left(\boldsymbol{n}_{\varepsilon} \cdot \operatorname{curl} \operatorname{curl} \overline{\boldsymbol{W}}_{\varepsilon}\right) .
\end{aligned}
$$

Since $\boldsymbol{n}_{\varepsilon} \cdot \operatorname{curl} \operatorname{curl} \overline{\boldsymbol{W}}_{\varepsilon}=\kappa^{2} \boldsymbol{n}_{\varepsilon} \cdot \overline{\boldsymbol{W}}_{\varepsilon}$, we get

$$
\begin{aligned}
\int_{\Gamma_{\text {meas }}}\left(\overline{\boldsymbol{E}_{\varepsilon}-\boldsymbol{E}_{\text {meas }}}\right) & \cdot \dot{\boldsymbol{E}}_{\varepsilon} d \mu=\int_{\partial B_{\varepsilon}(\mathbf{x})}\left(\kappa^{2}-\kappa^{2} \lambda^{2}\right)\left(\boldsymbol{n}_{\varepsilon} \times \boldsymbol{E}_{\varepsilon}\right) \cdot\left(\boldsymbol{n}_{\varepsilon} \times \overline{\boldsymbol{W}}_{\varepsilon}\right) \\
& +\int_{\partial B_{\varepsilon}(\mathbf{x})}-\left(\boldsymbol{n}_{\varepsilon} \cdot \operatorname{curl} \boldsymbol{E}_{\varepsilon}\right)\left(\boldsymbol{n}_{\varepsilon} \cdot \operatorname{curl} \overline{\boldsymbol{W}}_{\varepsilon}\right)-\kappa^{2}\left(\boldsymbol{n}_{\varepsilon} \cdot \boldsymbol{E}_{\varepsilon}\right)\left(\boldsymbol{n}_{\varepsilon} \cdot \overline{\boldsymbol{W}}_{\varepsilon}\right) .
\end{aligned}
$$

By (13), the result follows readily.

Theorem 5. For the transmission problem,

$$
\begin{aligned}
& \left.\frac{d}{d \tau} J\left(\mathbb{R}^{3} \backslash \Omega_{\varepsilon, \tau}\right)\right|_{\tau=0} \\
& =\operatorname{Re}\left[\int_{\partial B_{\varepsilon}(\mathbf{x})}\left(\beta_{e}^{2}-\beta_{i}^{2}\right)\left(\left(\boldsymbol{n}_{\varepsilon} \times \boldsymbol{E}_{\varepsilon}^{-}\right) \cdot\left(\boldsymbol{n}_{\varepsilon} \times \overline{\boldsymbol{W}}_{\varepsilon}^{-}\right)+\frac{\beta_{i}^{2}}{\beta_{e}^{2}}\left(\boldsymbol{n}_{\varepsilon} \cdot \boldsymbol{E}_{\varepsilon}^{-}\right)\left(\boldsymbol{n}_{\varepsilon} \cdot \overline{\boldsymbol{W}}_{\varepsilon}^{-}\right)\right)\right. \\
& \left.(21) \quad-\left(\alpha_{e}-\alpha_{i}\right)\left(\frac{\alpha_{i}}{\alpha_{e}}\left(\boldsymbol{n}_{\varepsilon} \times \operatorname{curl} \boldsymbol{E}_{\varepsilon}^{-}\right) \cdot\left(\boldsymbol{n}_{\varepsilon} \times \operatorname{curl} \overline{\boldsymbol{W}}_{\varepsilon}^{-}\right)+\left(\boldsymbol{n}_{\varepsilon} \cdot \operatorname{curl} \boldsymbol{E}_{\varepsilon}^{-}\right)\left(\boldsymbol{n}_{\varepsilon} \cdot \operatorname{curl} \overline{\boldsymbol{W}}_{\varepsilon}^{-}\right)\right)\right],
\end{aligned}
$$


where $\boldsymbol{E}_{\varepsilon}$ solves the forward problem (3) with $\Omega=B_{\varepsilon}(\mathbf{x})$ and $\overline{\boldsymbol{W}}_{\varepsilon}$ is the complex-conjugate of the solution $\boldsymbol{W}_{\varepsilon}$ of the associated adjoint problem; that is, it solves

$$
\begin{cases}\alpha_{e} \operatorname{curl} \operatorname{curl} \overline{\boldsymbol{W}}_{\varepsilon}-\beta_{e}^{2} \overline{\boldsymbol{W}}_{\varepsilon}=\left(\overline{\boldsymbol{E}_{\text {meas }}-\boldsymbol{E}_{\varepsilon}}\right) \delta_{\Gamma_{\text {meas }}} & \text { in } \mathbb{R}^{3} \backslash \overline{B_{\varepsilon}(\mathbf{x})}, \\ \alpha_{i} \operatorname{curl} \operatorname{curl} \overline{\boldsymbol{W}}_{\varepsilon}-\beta_{i}^{2} \overline{\boldsymbol{W}}_{\varepsilon}=\mathbf{0} & \text { in } B_{\varepsilon}(\mathbf{x}), \\ \boldsymbol{n}_{\varepsilon} \times \overline{\boldsymbol{W}}_{\varepsilon}^{-}-\boldsymbol{n}_{\varepsilon} \times \overline{\boldsymbol{W}}_{\varepsilon}^{+}=\mathbf{0} & \text { on } \partial B_{\varepsilon}(\mathbf{x}), \\ \alpha_{i} \boldsymbol{n}_{\varepsilon} \times \operatorname{curl} \overline{\boldsymbol{W}}_{\varepsilon}^{-}-\alpha_{e} \boldsymbol{n}_{\varepsilon} \times \operatorname{curl} \overline{\boldsymbol{W}}_{\varepsilon}^{+}=\mathbf{0} & \text { on } \partial B_{\varepsilon}(\mathbf{x}), \\ \lim _{|\mathbf{z}| \rightarrow \infty}|\mathbf{z}|\left|\operatorname{curl} \overline{\boldsymbol{W}}_{\varepsilon}(\mathbf{z}) \times \frac{\mathbf{z}}{|\mathbf{z}|}-i \kappa_{e} \overline{\boldsymbol{W}}_{\varepsilon}(\mathbf{z})\right|=0 . & \end{cases}
$$

Proof. The formula can be proved by working with the expression of $\dot{\boldsymbol{E}}_{\varepsilon}$ obtained in $[15,29]$ :

$$
\left\{\begin{array}{rlrl}
\alpha_{e} \operatorname{curl} \operatorname{curl} \dot{\boldsymbol{E}}_{\varepsilon}-\beta_{e}^{2} \dot{\boldsymbol{E}}_{\varepsilon}=\mathbf{0} & \text { in } \mathbb{R}^{3} \backslash \overline{B_{\varepsilon}(\mathbf{x})}, \\
\alpha_{i} \operatorname{curl} \operatorname{curl} \dot{\boldsymbol{E}}_{\varepsilon}-\beta_{i}^{2} \dot{\boldsymbol{E}}_{\varepsilon}=\mathbf{0} & \text { in } B_{\varepsilon}(\mathbf{x}), \\
\boldsymbol{n}_{\varepsilon} \times \dot{\boldsymbol{E}}_{\varepsilon}^{+}-\boldsymbol{n}_{\varepsilon} \times \dot{\boldsymbol{E}}_{\varepsilon}^{-}=-\boldsymbol{n}_{\varepsilon} \times\left(\left(\operatorname{curl} \boldsymbol{E}_{\varepsilon}^{+}-\operatorname{curl} \boldsymbol{E}_{\varepsilon}^{-}\right) \times \boldsymbol{n}_{\varepsilon}\right) & \\
& -\boldsymbol{n}_{\varepsilon} \times \boldsymbol{\nabla}\left(\boldsymbol{n}_{\varepsilon} \cdot\left(\boldsymbol{E}_{\varepsilon}^{+}-\boldsymbol{E}_{\varepsilon}^{-}\right)\right) & \\
\alpha_{e} \boldsymbol{n}_{\varepsilon} \times \operatorname{curl} \dot{\boldsymbol{E}}_{\varepsilon}^{+}-\alpha_{i} \boldsymbol{n}_{\varepsilon} \times \operatorname{curl} \dot{\boldsymbol{E}}_{\varepsilon}^{-}=-\boldsymbol{n}_{\varepsilon} \times\left(\left(\beta_{e}^{2} \boldsymbol{E}_{\varepsilon}^{+}-\beta_{i}^{2} \boldsymbol{E}_{\varepsilon}^{-}\right) \times \boldsymbol{n}_{\varepsilon}\right) & & \text { on } \partial B_{\varepsilon}(\mathbf{x}), \\
& -\boldsymbol{n}_{\varepsilon} \times \boldsymbol{\nabla}\left(\boldsymbol{n}_{\varepsilon} \cdot\left(\alpha_{e} \operatorname{curl} \boldsymbol{E}_{\varepsilon}^{+}-\alpha_{i} \operatorname{curl} \boldsymbol{E}_{\varepsilon}^{-}\right)\right) & \text {on } \partial B_{\varepsilon}(\mathbf{x}), \\
\lim _{|\mathbf{z}| \rightarrow \infty}|\mathbf{z}|\left|\operatorname{curl} \dot{\boldsymbol{E}}_{\varepsilon}(\mathbf{z}) \times \frac{\mathbf{z}}{|\mathbf{z}|}-i \kappa \dot{\boldsymbol{E}}_{\varepsilon}(\mathbf{z})\right|=0 . &
\end{array}\right.
$$

By Green's formulae we have

$$
\begin{aligned}
& \underbrace{\int_{B_{\varepsilon}(\mathbf{x})} \alpha_{i} \operatorname{curl} \operatorname{curl} \dot{\boldsymbol{E}}_{\varepsilon} \cdot \overline{\boldsymbol{W}}_{\varepsilon}-\beta_{i}^{2} \dot{\boldsymbol{E}}_{\varepsilon} \cdot \overline{\boldsymbol{W}}_{\varepsilon}}_{=0}-\alpha_{i} \int_{\partial B_{\varepsilon}(\mathbf{x})} \boldsymbol{n}_{\varepsilon} \times \operatorname{curl} \dot{\boldsymbol{E}}_{\varepsilon}^{-} \cdot \overline{\boldsymbol{W}_{\varepsilon}^{-}} \\
& =\underbrace{\int_{B_{\varepsilon}(\mathbf{x})} \alpha_{i} \operatorname{curl} \operatorname{curl} \overline{\boldsymbol{W}}_{\varepsilon} \cdot \dot{\boldsymbol{E}}_{\varepsilon}-\beta_{i}^{2} \dot{\boldsymbol{E}}_{\varepsilon} \cdot \overline{\boldsymbol{W}}_{\varepsilon}}_{=0}-\alpha_{i} \int_{\partial B_{\varepsilon}(\mathbf{x})} \boldsymbol{n}_{\varepsilon} \times \operatorname{curl} \overline{\boldsymbol{W}_{\varepsilon}^{-}} \cdot \dot{\boldsymbol{E}}_{\varepsilon}^{-}
\end{aligned}
$$

and

$$
\begin{aligned}
& \underbrace{\int_{B_{R} \backslash \overline{B_{\varepsilon}(\mathbf{x})}} \alpha_{e} \operatorname{curl} \operatorname{curl} \dot{\boldsymbol{E}}_{\varepsilon} \cdot \overline{\boldsymbol{W}}_{\varepsilon}-\beta_{e}^{2} \dot{\boldsymbol{E}}_{\varepsilon} \cdot \overline{\boldsymbol{W}}_{\varepsilon}}_{=0}+\alpha_{e} \int_{\partial B_{\varepsilon}(\mathbf{x})} \boldsymbol{n}_{\varepsilon} \times \operatorname{curl} \dot{\boldsymbol{E}}_{\varepsilon}^{+} \cdot \overline{\boldsymbol{W}_{\varepsilon}^{+}} \\
& \quad=\int_{B_{R} \backslash \overline{B_{\varepsilon}(\mathbf{x})}} \alpha_{e} \operatorname{curl} \operatorname{curl} \overline{\boldsymbol{W}}_{\varepsilon} \cdot \dot{\boldsymbol{E}}_{\varepsilon}-\beta_{e}^{2} \dot{\boldsymbol{E}}_{\varepsilon} \cdot \overline{\boldsymbol{W}}_{\varepsilon}+\alpha_{e} \int_{\partial B_{\varepsilon}(\mathbf{x})} \boldsymbol{n}_{\varepsilon} \times \operatorname{curl} \overline{\boldsymbol{W}_{\varepsilon}^{+}} \cdot \dot{\boldsymbol{E}}_{\varepsilon}^{+} .
\end{aligned}
$$

Applying now the surface diverge operator to the transmission conditions and [43, eq. (2.5.203)], we find that

$$
0=\operatorname{div}_{\partial B_{\varepsilon}(\mathbf{x})}\left(\boldsymbol{n}_{\varepsilon} \times \mathbf{U}^{-}-\boldsymbol{n}_{\varepsilon} \times \mathbf{U}^{+}\right)=-\boldsymbol{n}_{\varepsilon} \cdot \operatorname{curl} \mathbf{U}^{-}+\boldsymbol{n}_{\varepsilon} \cdot \operatorname{curl} \mathbf{U}^{+}
$$


and

$$
\begin{aligned}
0 & =\operatorname{div}_{\partial B_{\varepsilon}(\mathbf{x})}\left(\alpha_{i} \boldsymbol{n}_{\varepsilon} \times \operatorname{curl} \mathbf{U}^{-}-\alpha_{e} \boldsymbol{n}_{\varepsilon} \times \operatorname{curl} \mathbf{U}^{+}\right) \\
& =-\alpha_{i} \boldsymbol{n}_{\varepsilon} \cdot \operatorname{curl} \operatorname{curl} \mathbf{U}^{-}+\alpha_{e} \boldsymbol{n}_{\varepsilon} \cdot \operatorname{curl} \operatorname{curl} \mathbf{U}^{+}=-\beta_{i}^{2} \boldsymbol{n}_{\varepsilon} \cdot \mathbf{U}^{-}+\beta_{e}^{2} \boldsymbol{n}_{\varepsilon} \cdot \mathbf{U}^{+}
\end{aligned}
$$

where $\mathbf{U}$ is either $\boldsymbol{E}_{\varepsilon}$ or $\overline{\boldsymbol{W}}_{\varepsilon}$. Then, the following relations for the normal components on $\partial B_{\varepsilon}(\mathbf{x})$ follow readily:

$$
\begin{array}{ll}
\boldsymbol{n}_{\varepsilon} \cdot \boldsymbol{E}_{\varepsilon}^{+}=\frac{\beta_{i}^{2}}{\beta_{e}^{2}} \boldsymbol{n}_{\varepsilon} \cdot \boldsymbol{E}_{\varepsilon}^{-}, & \boldsymbol{n}_{\varepsilon} \cdot \overline{\boldsymbol{W}_{\varepsilon}^{+}}=\frac{\beta_{i}^{2}}{\beta_{e}^{2}} \boldsymbol{n}_{\varepsilon} \cdot \overline{\boldsymbol{W}_{\varepsilon}^{-}} \\
\boldsymbol{n}_{\varepsilon} \cdot \operatorname{curl} \boldsymbol{E}_{\varepsilon}^{+}=\boldsymbol{n}_{\varepsilon} \cdot \operatorname{curl} \boldsymbol{E}_{\varepsilon}^{-}, & \boldsymbol{n}_{\varepsilon} \cdot \operatorname{curl} \overline{\boldsymbol{W}_{\varepsilon}^{+}}=\boldsymbol{n}_{\varepsilon} \cdot \operatorname{curl} \overline{\boldsymbol{W}_{\varepsilon}^{-}}
\end{array}
$$

Finally,

$$
\begin{aligned}
\int_{\Gamma_{\text {meas }}}\left(\overline{\boldsymbol{E}_{\text {meas }}-\boldsymbol{E}_{\varepsilon}}\right) \cdot \dot{\boldsymbol{E}}_{\varepsilon} d \mu= & \int_{\partial B_{\varepsilon}(\mathbf{x})}\left(\alpha_{e} \boldsymbol{n}_{\varepsilon} \times \operatorname{curl} \dot{\boldsymbol{E}}_{\varepsilon}^{+}-\alpha_{i} \boldsymbol{n}_{\varepsilon} \times \operatorname{curl} \dot{\boldsymbol{E}}_{\varepsilon}^{-}\right) \cdot \overline{\boldsymbol{W}}_{\varepsilon} \\
& +\alpha_{i} \int_{\partial B_{\varepsilon}(\mathbf{x})} \operatorname{curl} \overline{\boldsymbol{W}}_{\varepsilon} \cdot\left(\boldsymbol{n}_{\varepsilon} \times\left(\dot{\boldsymbol{E}}_{\varepsilon}^{+}-\dot{\boldsymbol{E}}_{\varepsilon}^{-}\right)\right) \\
= & \int_{\partial B_{\varepsilon}(\mathbf{x})}-\left(\beta_{e}^{2}-\beta_{i}^{2}\right)\left(\boldsymbol{n}_{\varepsilon} \times \boldsymbol{E}_{\varepsilon}^{-}\right) \cdot\left(\boldsymbol{n}_{\varepsilon} \times \overline{\boldsymbol{W}}_{\varepsilon}\right) \\
& +\int_{\partial B_{\varepsilon}(\mathbf{x})}\left(\alpha_{e}-\alpha_{i}\right)\left(\boldsymbol{n}_{\varepsilon} \cdot \operatorname{curl} \boldsymbol{E}_{\varepsilon}^{-}\right)\left(\boldsymbol{n}_{\varepsilon} \cdot \operatorname{curl} \overline{\boldsymbol{W}_{\varepsilon}^{-}}\right) \\
& +\int_{\partial B_{\varepsilon}(\mathbf{x})}-\left(\alpha_{i}-\alpha_{e}\right) \frac{\alpha_{i}}{\alpha_{e}}\left(\boldsymbol{n}_{\varepsilon} \times \operatorname{curl} \boldsymbol{E}_{\varepsilon}^{-}\right) \cdot\left(\boldsymbol{n}_{\varepsilon} \times \operatorname{curl} \overline{\boldsymbol{W}_{\varepsilon}^{-}}\right) \\
& +\int_{\partial B_{\varepsilon}(\mathbf{x})}\left(\beta_{i}^{2}-\beta_{e}^{2}\right) \frac{\beta_{i}^{2}}{\beta_{e}^{2}}\left(\boldsymbol{n}_{\varepsilon} \cdot \boldsymbol{E}_{\varepsilon}^{-}\right)\left(\boldsymbol{n}_{\varepsilon} \cdot \overline{\boldsymbol{W}_{\varepsilon}^{-}}\right),
\end{aligned}
$$

which finishes the result thanks to (13).

Remark 1. Setting $\beta_{e}=\kappa, \alpha_{e}=1, \beta_{i}=0$, and $\alpha_{i}=0$ in (21), we recover (20) for the Neumann case.

The formulae for the TD will be obtained now by using Mie series expansions [41, Chapter 9]. Let $\mathbf{x} \in \Gamma_{\text {meas. }}$. The incident field $\boldsymbol{E}^{\text {inc }}$ that satisfies the second order Maxwell equation in the absence of electromagnetic cavities can be written for any $\mathbf{z}$ inside $\Gamma_{\text {meas }}$ as

$$
\boldsymbol{E}^{\mathrm{inc}}(\mathbf{z})=\sum_{n=1}^{\infty} \sum_{j=-n}^{n}\left[\alpha_{n, j}^{(1)}(\mathbf{x}) M_{n, j}^{(1)}(\kappa, \mathbf{z}-\mathbf{x})+\beta_{n, j}^{(1)}(\mathbf{x}) N_{n, j}^{(1)}(\kappa, \mathbf{z}-\mathbf{x})\right],
$$

where $\left(\alpha_{n, j}^{(1)}\right)$ and $\left(\beta_{n, j}^{(1)}\right)$ are complex-valued sequences depending on $\mathbf{x}$ but independent of $\mathbf{z}$, and for $\varepsilon>0$ and $\boldsymbol{\xi} \in \mathbb{S}^{2}$ we have

$$
\begin{aligned}
M_{n, j}^{(1)}(\kappa, \varepsilon \boldsymbol{\xi}) & =j_{n}(\kappa \varepsilon) \operatorname{curl}_{\mathbb{S}^{2}} Y_{n}^{j}(\boldsymbol{\xi}), \\
N_{n, j}^{(1)}(\kappa, \varepsilon \boldsymbol{\xi}) & =\frac{\boldsymbol{\xi}}{i \kappa \varepsilon} n(n+1) j_{n}(\kappa \varepsilon) Y_{n}^{j}(\boldsymbol{\xi})+\frac{1}{i \kappa \varepsilon}\left(j_{n}(\kappa \varepsilon)+\kappa \varepsilon j_{n}^{\prime}(\kappa \varepsilon)\right) \nabla_{\mathbb{S}^{2}} Y_{n}^{j}(\boldsymbol{\xi}) .
\end{aligned}
$$


Indeed, in the case of an incident plane wave $\boldsymbol{E}^{\text {inc }}(\mathbf{z})=\boldsymbol{p} e^{i \kappa \mathbf{z} \cdot \boldsymbol{d}}$ with $\boldsymbol{d}, \boldsymbol{p} \in \mathbb{S}^{2}$ and $\boldsymbol{d} \cdot \boldsymbol{p}=0$,

$$
\alpha_{n, j}^{(1)}(\mathbf{x})=\frac{4 \pi i^{n} e^{i \kappa \mathbf{x} \cdot \boldsymbol{d}}}{n(n+1)} \operatorname{curl}_{\mathbb{S}^{2}} \overline{Y_{n}^{j}(\boldsymbol{d})} \cdot \boldsymbol{p}, \quad \beta_{n, j}^{(1)}(\mathbf{x})=\frac{4 \pi i^{n} e^{i \kappa \mathbf{x} \cdot \boldsymbol{d}}}{n(n+1)} \nabla_{\mathbb{S}^{2}} \overline{Y_{n}^{j}(\boldsymbol{d})} \cdot \boldsymbol{p} .
$$

In the more general case, we use the addition theorem for vector spherical harmonics [38], which links $M_{n, j}^{(1)}(\kappa, \mathbf{z})$ or $N_{n, j}^{(1)}(\kappa, \mathbf{z})$ to $M_{n, j}^{(1)}(\kappa, \mathbf{z}-\mathbf{x})$ and $M_{n, j}^{(1)}(\kappa, \mathbf{z}-\mathbf{x})$. This means that the coefficients $\alpha_{n, j}^{(1)}(\mathbf{x})$ and $\beta_{n, j}^{(1)}(\mathbf{x})$ can be expressed in terms of the sequences $\left(\alpha_{n, j}^{(1)}(\mathbf{0})\right)_{n, j}$ and $\left(\beta_{n, j}^{(1)}(\mathbf{0})\right)_{n, j}$.

Proposition 6. Let $\boldsymbol{\xi} \in \mathbb{S}^{2}$ and $\mathbf{y}_{\text {obs }} \in \Gamma_{\text {meas. }}$. For the impedance problem, we have

$$
\begin{aligned}
& \lim _{\varepsilon \rightarrow 0} \boldsymbol{E}_{\varepsilon}\left(\mathbf{y}_{\mathrm{obs}}\right)=\boldsymbol{E}^{\mathrm{inc}}\left(\mathbf{y}_{\mathrm{obs}}\right), \\
& \lim _{\varepsilon \rightarrow 0} \boldsymbol{n}_{\varepsilon} \times \boldsymbol{E}_{\varepsilon}(\mathbf{x}+\varepsilon \boldsymbol{\xi})=\frac{3}{2} \delta_{0}(\lambda) \boldsymbol{\xi} \times \boldsymbol{E}^{\mathrm{inc}}(\mathbf{x}), \\
& \lim _{\varepsilon \rightarrow 0} \boldsymbol{n}_{\varepsilon} \cdot \operatorname{curl} \boldsymbol{E}_{\varepsilon}(\mathbf{x}+\varepsilon \boldsymbol{\xi})=3 \boldsymbol{\xi} \cdot \mathbf{c u r l} \boldsymbol{E}^{\mathrm{inc}}(\mathbf{x}), \\
& \lim _{\varepsilon \rightarrow 0} \boldsymbol{n}_{\varepsilon} \cdot \boldsymbol{E}_{\varepsilon}(\mathbf{x}+\varepsilon \boldsymbol{\xi})=3 \boldsymbol{\xi} \cdot \boldsymbol{E}^{\mathrm{inc}}(\mathbf{x}) \quad \text { if } \lambda \neq 0,
\end{aligned}
$$

where $\delta_{0}(\lambda)=1$ if $\lambda=0$ and $\delta_{0}(\lambda)=0$ otherwise.

Proof. Using the Mie series expansion, we find that

$$
\boldsymbol{E}_{\varepsilon}(\mathbf{z})-\boldsymbol{E}^{\mathrm{inc}}(\mathbf{z})=-\sum_{n=1}^{\infty} \sum_{j=-n}^{n}\left[\alpha_{n, j}^{(3)}(\mathbf{x}, \varepsilon) M_{n, j}^{(3)}(\kappa, \mathbf{z}-\mathbf{x})+\beta_{n, j}^{(3)}(\mathbf{x}, \varepsilon) N_{n, j}^{(3)}(\kappa, \mathbf{z}-\mathbf{x})\right],
$$

where

$$
\begin{aligned}
& \alpha_{n, j}^{(3)}(\mathbf{x}, \varepsilon)=\frac{(1+i \lambda \kappa \varepsilon) j_{n}(\kappa \varepsilon)+\kappa \varepsilon j_{n}^{\prime}(\kappa \varepsilon)}{(1+i \kappa \lambda \varepsilon) h_{n}^{(1)}(\kappa \varepsilon)+\kappa \varepsilon h_{n}^{(1)^{\prime}}(\kappa \varepsilon)} \alpha_{n, j}^{(1)}(\mathbf{x}), \\
& \beta_{n, j}^{(3)}(\mathbf{x}, \varepsilon)=\frac{\left(-\kappa^{2} \varepsilon+i \kappa \lambda\right) j_{n}(\kappa \varepsilon)+i \lambda \kappa^{2} \varepsilon j_{n}^{\prime}(\kappa \varepsilon)}{\left(-\kappa^{2} \varepsilon+i \kappa \lambda\right) h_{n}^{(1)}(\kappa \varepsilon)+i \lambda \kappa^{2} \varepsilon h_{n}^{(1)^{\prime}}(\kappa \varepsilon)} \beta_{n, j}^{(1)}(\mathbf{x}),
\end{aligned}
$$

and

$$
\begin{aligned}
M_{n, j}^{(3)}(\kappa, \varepsilon \boldsymbol{\xi}) & =h_{n}^{(1)}(\kappa \varepsilon) \operatorname{curl}_{\mathbb{S}^{2}} Y_{n}^{j}(\boldsymbol{\xi}), \\
N_{n, j}^{(3)}(\kappa, \varepsilon \boldsymbol{\xi}) & =\frac{\boldsymbol{\xi}}{i \kappa \varepsilon} n(n+1) h_{n}^{(1)}(\kappa \varepsilon) Y_{n}^{j}(\boldsymbol{\xi})+\frac{1}{i \kappa \varepsilon}\left(h_{n}^{(1)}(\kappa \varepsilon)+\kappa \varepsilon h_{n}^{(1)^{\prime}}(\kappa \varepsilon)\right) \boldsymbol{\nabla}_{\mathbb{S}^{2}} Y_{n}^{j}(\boldsymbol{\xi}) .
\end{aligned}
$$

If $\mathbf{z}=\mathbf{x}+\varepsilon \boldsymbol{\xi} \in \partial B_{\varepsilon}(\mathbf{x})$ with $\boldsymbol{\xi} \in \mathbb{S}^{2}$, then

$$
\begin{aligned}
\boldsymbol{n}_{\varepsilon}(\mathbf{z}) \times \boldsymbol{E}_{\varepsilon}(\mathbf{z}) & =\sum_{n=1}^{\infty} \sum_{j=-n}^{n}\left[\zeta_{n}(\varepsilon) \alpha_{n, j}^{(1)}(\mathbf{x}) \boldsymbol{\xi} \times M_{n, j}^{(1)}(\kappa, \varepsilon \boldsymbol{\xi})+\gamma_{n}(\varepsilon) \beta_{n, j}^{(1)}(\mathbf{x}) \boldsymbol{\xi} \times N_{n, j}^{(1)}(\kappa, \varepsilon \boldsymbol{\xi})\right], \\
\boldsymbol{n}_{\varepsilon}(\mathbf{z}) \cdot \boldsymbol{E}_{\varepsilon}(\mathbf{z}) & =\sum_{n=1}^{\infty} \sum_{j=-n}^{n}\left[\chi_{n}(\varepsilon) \beta_{n, j}^{(1)}(\mathbf{x}) \boldsymbol{\xi} \cdot N_{n, j}^{(1)}(\kappa, \varepsilon \boldsymbol{\xi})\right]
\end{aligned}
$$


with

$$
\begin{aligned}
& \zeta_{n}(\varepsilon)=1-\frac{h_{n}^{(1)}(\kappa \varepsilon)}{j_{n}(\kappa \varepsilon)} \cdot \frac{(1+i \kappa \lambda \varepsilon) j_{n}(\kappa \varepsilon)+\kappa \varepsilon j_{n}^{\prime}(\kappa \varepsilon)}{(1+i \kappa \lambda \varepsilon) h_{n}^{(1)}(\kappa \varepsilon)+\kappa \varepsilon h_{n}^{(1)^{\prime}}(\kappa \varepsilon)}, \\
& \gamma_{n}(\varepsilon)=1-\frac{h_{n}^{(1)}(\kappa \varepsilon)+\kappa \varepsilon h_{n}^{(1)^{\prime}}(\kappa \varepsilon)}{j_{n}(\kappa \varepsilon)+\kappa \varepsilon j_{n}^{\prime}(\kappa \varepsilon)} \cdot \frac{\left(-\kappa^{2} \varepsilon+i \kappa \lambda\right) j_{n}(\kappa \varepsilon)+i \lambda \kappa^{2} \varepsilon j_{n}^{\prime}(\kappa \varepsilon)}{\left(-\kappa^{2} \varepsilon+i \kappa \lambda\right) h_{n}^{(1)}(\kappa \varepsilon)+i \lambda \kappa^{2} \varepsilon h_{n}^{(1)^{\prime}}(\kappa \varepsilon)} \\
& \chi_{n}(\varepsilon)=1-\frac{h_{n}^{(1)}(\kappa \varepsilon)}{j_{n}(\kappa \varepsilon)} \cdot \frac{\left(-\kappa^{2} \varepsilon+i \kappa \lambda\right) j_{n}(\kappa \varepsilon)+i \lambda \kappa^{2} \varepsilon j_{n}^{\prime}(\kappa \varepsilon)}{\left(-\kappa^{2} \varepsilon+i \kappa \lambda\right) h_{n}^{(1)}(\kappa \varepsilon)+i \lambda \kappa^{2} \varepsilon h_{n}^{(1)^{\prime}}(\kappa \varepsilon)} .
\end{aligned}
$$

We have $j_{n}(\kappa \varepsilon) \underset{\varepsilon \rightarrow 0}{\approx}(\kappa \varepsilon)^{n} \frac{2^{n}(n !)}{(2 n+1) !}, y_{n}(\kappa \varepsilon) \underset{\varepsilon \rightarrow 0}{\approx}-(\kappa \varepsilon)^{-(n+1)} \frac{(2 n-1) !}{2^{n-1}(n-1) !}[1$, formulae (10.1.4) and (10.1.5)] and $h_{n}^{(1)}(\kappa \varepsilon)=j_{n}(\kappa \varepsilon)+i y_{n}(\kappa \varepsilon)$. For the derivatives we use, when $z_{n}=j_{n}, h_{n}^{(1)}$,

$$
z_{n}^{\prime}(\kappa \varepsilon)=-z_{n+1}(\kappa \varepsilon)+\frac{n}{\kappa \varepsilon} z_{n}(\kappa \varepsilon) .
$$

It follows that

$$
\begin{aligned}
& M_{n, j}^{(1)}(\kappa, \varepsilon \boldsymbol{\xi})=O\left(\varepsilon^{n}\right), \\
& N_{n, j}^{(1)}(\kappa, \varepsilon \boldsymbol{\xi})=-i(\kappa \varepsilon)^{n-1}\left\{\frac{n(n+1) 2^{n}(n !)}{(2 n+1) !} \boldsymbol{\xi} Y_{n}^{j}(\boldsymbol{\xi})+\frac{(n+1) 2^{n}(n !)}{(2 n+1) !} \nabla_{\mathbb{S}^{2}} Y_{n}^{j}(\boldsymbol{\xi})\right\}+O\left(\varepsilon^{n}\right),
\end{aligned}
$$

and

$$
\zeta_{n}(\varepsilon) \underset{\varepsilon \rightarrow 0}{\approx} 1+\frac{n+1}{n}, \quad \gamma_{n}(\varepsilon) \underset{\varepsilon \rightarrow 0}{\approx}\left\{\begin{array}{cc}
1+\frac{n}{n+1} & \text { if } \lambda=0 \\
O(\varepsilon) & \text { if } \lambda \neq 0
\end{array}\right.
$$

and when $\lambda \neq 0$, we have $\chi_{n}(\varepsilon) \underset{\varepsilon \rightarrow 0}{\approx} 1+\frac{n+1}{n}$. From (23), we deduce that

$$
\boldsymbol{n}_{\varepsilon}(\mathbf{z}) \times \boldsymbol{E}^{\mathrm{inc}}(\mathbf{z})=\sum_{j=-1}^{1} \beta_{1, j}(\mathbf{x}) \boldsymbol{\xi} \times N_{1, j}^{(1)}(\kappa, \varepsilon \boldsymbol{\xi})+O(\varepsilon)
$$

and

$$
\begin{aligned}
\boldsymbol{n}_{\varepsilon}(\mathbf{z}) \times \boldsymbol{E}_{\varepsilon}(\mathbf{z}) & =\gamma_{1}(\varepsilon) \sum_{j=-1}^{1} \beta_{1, j}^{(1)}(\mathbf{x}) \boldsymbol{\xi} \times N_{1, j}^{(1)}(\kappa, \varepsilon \boldsymbol{\xi})+O(\varepsilon) \\
& =\frac{3}{2} \delta_{0}(\lambda) \sum_{j=-1}^{1} \beta_{1, j}^{(1)}(\mathbf{x}) \boldsymbol{\xi} \times N_{1, j}^{(1)}(\kappa, \varepsilon \boldsymbol{\xi})+O(\varepsilon)=\frac{3}{2} \delta_{0}(\lambda) \boldsymbol{\xi} \times \boldsymbol{E}^{\mathrm{inc}}(\mathbf{x})+O(\varepsilon) .
\end{aligned}
$$

In addition,

$$
\boldsymbol{n}_{\varepsilon}(\mathbf{z}) \cdot \boldsymbol{E}_{\varepsilon}(\mathbf{z})=\chi_{1}(\varepsilon) \sum_{j=-1}^{1} \beta_{1, j}^{(1)}(\mathbf{x}) \boldsymbol{\xi} \cdot N_{1, j}^{(1)}(\kappa, \varepsilon \boldsymbol{\xi})+O(\varepsilon)=3 \boldsymbol{\xi} \cdot \boldsymbol{E}^{\mathrm{inc}}(\mathbf{x})+O(\varepsilon) .
$$


We also have

$$
\begin{array}{r}
\operatorname{curl} \boldsymbol{E}^{\mathrm{inc}}(\mathbf{z})=i \kappa \sum_{n=1}^{\infty} \sum_{j=-n}^{n}\left[\alpha_{n, j}^{(1)}(\mathbf{x}) N_{n, j}^{(1)}(\kappa, \mathbf{z}-\mathbf{x})-\beta_{n, j}^{(1)}(\mathbf{x}) M_{n, j}^{(1)}(\kappa, \mathbf{z}-\mathbf{x})\right], \\
\operatorname{curl} \boldsymbol{E}_{\varepsilon}(\mathbf{z})-\operatorname{curl} \boldsymbol{E}^{\mathrm{inc}}(\mathbf{z})=-i \kappa \sum_{n=1}^{\infty} \sum_{j=-n}^{n}\left[\alpha_{n, j}^{(3)}(\mathbf{x}) N_{n, j}^{(3)}(\kappa, \mathbf{z}-\mathbf{x})-\beta_{n, j}^{(3)}(\mathbf{x}) M_{n, j}^{(3)}(\kappa, \mathbf{z}-\mathbf{x})\right],
\end{array}
$$

since $\operatorname{curl} M_{n, j}^{(a)}=i \kappa N_{n, j}^{(a)}$ and $\operatorname{curl} N_{n, j}^{(a)}=-i \kappa M_{n, j}^{(a)}$ for $a=1,3$. We deduce that

$$
\begin{gathered}
\boldsymbol{n}_{\varepsilon}(\mathbf{z}) \cdot \operatorname{curl} \boldsymbol{E}^{\mathrm{inc}}(\mathbf{z})=i \kappa \sum_{j=-1}^{1} \alpha_{n, j}^{(1)}(\mathbf{x}) \boldsymbol{\xi} \cdot N_{1, j}^{(1)}(\kappa, \varepsilon \boldsymbol{\xi})+O(\varepsilon), \\
\boldsymbol{n}_{\varepsilon}(\mathbf{z}) \cdot \operatorname{curl} \boldsymbol{E}_{\varepsilon}(\mathbf{z})=\zeta_{1}(\varepsilon) i \kappa \sum_{j=-1}^{1} \alpha_{1, j}^{(1)}(\mathbf{x}) \boldsymbol{\xi} \cdot N_{1, j}^{(1)}(\kappa, \varepsilon \boldsymbol{\xi})+O(\varepsilon)=3 \boldsymbol{\xi} \cdot \operatorname{curl} \boldsymbol{E}^{\text {inc }}(\mathbf{x})+O(\varepsilon) .
\end{gathered}
$$

Finally, we have for any $n \geq 1$ and $|j| \leq n$ that $\alpha_{n, j}^{(3)}(\mathbf{x}, \varepsilon) \underset{\varepsilon \rightarrow 0}{\rightarrow} 0$ and $\beta_{n, j}^{(3)}(\mathbf{x}, \varepsilon) \underset{\varepsilon \rightarrow 0}{\rightarrow} 0$, which leads to $\boldsymbol{E}_{\varepsilon}\left(\mathbf{y}_{\text {obs }}\right)-\boldsymbol{E}^{\text {inc }}\left(\mathbf{y}_{\text {obs }}\right) \underset{\varepsilon \rightarrow 0}{\rightarrow} \mathbf{0}$.

We now derive analogous expressions for $\overline{\boldsymbol{W}}_{\varepsilon}$. Let us introduce first the solution $\overline{\boldsymbol{W}}_{\varepsilon}^{\text {inc }}$ to the adjoint problem (19) in the absence of defects, i.e.,

$$
\left\{\begin{array}{c}
\operatorname{curl} \operatorname{curl} \overline{\boldsymbol{W}}_{\varepsilon}^{\mathrm{inc}}-\kappa^{2} \overline{\boldsymbol{W}}_{\varepsilon}^{\mathrm{inc}}=\left(\overline{\boldsymbol{E}_{\text {meas }}-\boldsymbol{E}_{\varepsilon}}\right) \delta_{\Gamma_{\text {meas }}} \quad \text { in } \mathbb{R}^{3} \\
\lim _{|\mathbf{z}| \rightarrow \infty}|\mathbf{z}|\left|\operatorname{curl} \overline{\boldsymbol{W}}_{\varepsilon}^{\mathrm{inc}}(\mathbf{z}) \times \frac{\mathbf{z}}{|\mathbf{z}|}-i \kappa \overline{\boldsymbol{W}}_{\varepsilon}^{\mathrm{inc}}(\mathbf{z})\right|=0,
\end{array}\right.
$$

where $\boldsymbol{E}_{\varepsilon}$ solves the forward problem (2) with $\Omega=B_{\varepsilon}(\mathbf{x})$. Let set $G(\kappa, \mathbf{z})=\frac{e^{i \kappa|\mathbf{z}|}}{4 \pi|\mathbf{z}|}$. We know that

$$
\overline{\boldsymbol{W}}_{\varepsilon}^{\text {inc }}(\mathbf{z})=\frac{1}{\kappa^{2}} \operatorname{curl}^{\mathrm{curl}} \mathbf{z}^{\mathbf{z}}\left\{\int_{\Gamma_{\text {meas }}} G\left(\kappa, \mathbf{z}-\mathbf{y}_{\text {obs }}\right)\left(\overline{\boldsymbol{E}_{\text {meas }}\left(\mathbf{y}_{\text {obs }}\right)-\boldsymbol{E}_{\varepsilon}\left(\mathbf{y}_{\text {obs }}\right)}\right) d \mu\left(\mathbf{y}_{\text {obs }}\right)\right\} .
$$

When $\varepsilon=0$, we have

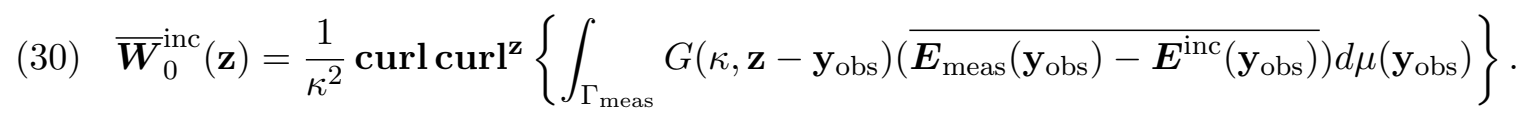

Proposition 7. Let $\boldsymbol{\xi} \in \mathbb{S}^{2}$. For the impedance problem, we have

$$
\begin{aligned}
& \lim _{\varepsilon \rightarrow 0} \overline{\boldsymbol{W}}_{\varepsilon}^{\mathrm{inc}}(\mathbf{x})=\overline{\boldsymbol{W}}_{0}^{\mathrm{inc}}(\mathbf{x}), \\
& \lim _{\varepsilon \rightarrow 0} \boldsymbol{n}_{\varepsilon} \times \overline{\boldsymbol{W}}_{\varepsilon}(\mathbf{x}+\varepsilon \boldsymbol{\xi})=\frac{3}{2} \delta_{0}(\lambda) \boldsymbol{\xi} \times \overline{\boldsymbol{W}}_{0}^{\mathrm{inc}}(\mathbf{x}), \\
& \lim _{\varepsilon \rightarrow 0} \boldsymbol{n}_{\varepsilon} \cdot \operatorname{curl} \overline{\boldsymbol{W}}_{\varepsilon}(\mathbf{x}+\varepsilon \boldsymbol{\xi})=3 \boldsymbol{\xi} \cdot \operatorname{curl} \overline{\boldsymbol{W}}_{0}^{\mathrm{inc}}(\mathbf{x}) \quad \text { if } \lambda \neq 0,
\end{aligned}
$$

where $\delta_{0}(\lambda)=1$ if $\lambda=0$ and $\delta_{0}(\lambda)=0$ otherwise. 
Proof. The result (31) is deduced from (24). The solution to the adjoint problem (19) can be written as $\overline{\boldsymbol{W}}_{\varepsilon}=\overline{\boldsymbol{W}}_{\varepsilon}^{\text {scat }}+\overline{\boldsymbol{W}}_{\varepsilon}^{\text {inc }}$, where $\overline{\boldsymbol{W}}_{\varepsilon}^{\text {scat }}$ solves the following scattering problem:

$$
\begin{cases}\operatorname{curl} \operatorname{curl} \overline{\boldsymbol{W}}_{\varepsilon}^{\text {scat }}-\kappa^{2} \overline{\boldsymbol{W}}_{\varepsilon}^{\text {scat }}=\mathbf{0} & \text { in } \mathbb{R}^{3} \backslash \overline{B_{\varepsilon}(\mathbf{x})}, \\ \boldsymbol{n}_{\varepsilon} \times \operatorname{curl}\left(\overline{\boldsymbol{W}}_{\varepsilon}^{\text {scat }}+\overline{\boldsymbol{W}}_{\varepsilon}^{\text {inc }}\right)-i \kappa \lambda \boldsymbol{n}_{\varepsilon} \times\left(\overline{\boldsymbol{W}}_{\varepsilon}^{\text {scat }}+\overline{\boldsymbol{W}}_{\varepsilon}^{\text {inc }}\right) \times \boldsymbol{n}_{\varepsilon}=\mathbf{0} & \text { on } \partial B_{\varepsilon}(\mathbf{x}), \\ \lim _{|\mathbf{z}| \rightarrow \infty}|\mathbf{z}|\left|\operatorname{curl} \overline{\boldsymbol{W}}_{\varepsilon}^{\text {scat }}(\mathbf{z}) \times \frac{\mathbf{z}}{|\mathbf{z}|}-i \kappa \overline{\boldsymbol{W}}_{\varepsilon}^{\text {scat }}(\mathbf{z})\right|=0 . & \end{cases}
$$

Using the Mie series expansion of the fundamental solution, we get, when $|\mathbf{z}-\mathbf{x}|<\left|\mathbf{y}_{\text {obs }}-\mathbf{x}\right|$ (and in particular when $\mathbf{z} \in \partial B_{\varepsilon}(\mathbf{x})$ ),

$$
\overline{\boldsymbol{W}}_{\varepsilon}^{\mathrm{inc}}(\mathbf{z})=\sum_{n=1}^{\infty} \sum_{j=-n}^{n}\left[\alpha_{n, j}^{(1)}(\mathbf{x}, \varepsilon) M_{n, j}^{(1)}(\kappa, \mathbf{z}-\mathbf{x})+\beta_{n, j}^{(1)}(\mathbf{x}, \varepsilon) N_{n, j}^{(1)}(\kappa, \mathbf{z}-\mathbf{x})\right]
$$

with

$$
\alpha_{n, j}^{(1)}(\mathbf{x}, \varepsilon)=-\frac{i \kappa(-1)^{j}}{n(n+1)} \int_{\Gamma_{\text {meas }}} M_{n,-j}^{(3)}\left(\kappa, \mathbf{y}_{\text {obs }}-\mathbf{x}\right) \cdot\left(\overline{\boldsymbol{E}_{\varepsilon}\left(\mathbf{y}_{\text {obs }}\right)-\boldsymbol{E}_{\text {meas }}\left(\mathbf{y}_{\text {obs }}\right)}\right) d \mu\left(\mathbf{y}_{\text {obs }}\right)
$$

and

$$
\beta_{n, j}^{(1)}(\mathbf{x}, \varepsilon)=\frac{i \kappa(-1)^{j}}{n(n+1)} \int_{\Gamma_{\text {meas }}} N_{n,-j}^{(3)}\left(\kappa, \mathbf{y}_{\text {obs }}-\mathbf{x}\right) \cdot\left(\overline{\boldsymbol{E}_{\varepsilon}\left(\mathbf{y}_{\text {obs }}\right)-\boldsymbol{E}_{\text {meas }}\left(\mathbf{y}_{\text {obs }}\right)}\right) d \mu\left(\mathbf{y}_{\text {obs }}\right) .
$$

Then $\overline{\boldsymbol{W}}_{\varepsilon}^{\text {scat }}$ can be expressed in terms of the Mie series (28), and following the proof of (19) in Theorem 4, we immediately deduce (32) and (33).

Proposition 8. Let $\boldsymbol{\xi} \in \mathbb{S}^{2}$. For the Dirichlet problem, we have

$$
\begin{aligned}
& \lim _{\varepsilon \rightarrow 0} \boldsymbol{n}_{\varepsilon} \times \operatorname{curl} \boldsymbol{E}_{\varepsilon}(\mathbf{x}+\varepsilon \boldsymbol{\xi})=\frac{3}{2} \boldsymbol{\xi} \times \operatorname{curl} \boldsymbol{E}^{\mathrm{inc}}(\mathbf{x}), \\
& \lim _{\varepsilon \rightarrow 0} \boldsymbol{n}_{\varepsilon} \cdot \boldsymbol{E}_{\varepsilon}(\mathbf{x}+\varepsilon \boldsymbol{\xi})=3 \boldsymbol{\xi} \cdot \boldsymbol{E}^{\mathrm{inc}}(\mathbf{x}),
\end{aligned}
$$

and $\overline{\boldsymbol{W}}_{\varepsilon}$ has the same asymptotic behavior as $\boldsymbol{E}_{\varepsilon}$ with $\boldsymbol{E}^{\text {inc }}$ replaced by $\overline{\boldsymbol{W}}_{0}^{\text {inc }}$ as given by (30).

Proof. Using the Mie series expansion, we find that

$$
\boldsymbol{E}_{\varepsilon}(\mathbf{z})-\boldsymbol{E}^{\mathrm{inc}}(\mathbf{z})=-\sum_{n=1}^{\infty} \sum_{j=-n}^{n}\left[\alpha_{n, j}^{(3)}(\mathbf{x}, \varepsilon) M_{n, j}^{(3)}(\kappa, \mathbf{z}-\mathbf{x})+\beta_{n, j}^{(3)}(\mathbf{x}, \varepsilon) N_{n, j}^{(3)}(\kappa, \mathbf{z}-\mathbf{x})\right],
$$

where

$$
\alpha_{n, j}^{(3)}(\mathbf{x}, \varepsilon)=\frac{j_{n}(\kappa \varepsilon)}{h_{n}^{(1)}(\kappa \varepsilon)} \alpha_{n, j}^{(1)}(\mathbf{x}), \quad \beta_{n, j}^{(3)}(\mathbf{x}, \varepsilon)=\frac{j_{n}(\kappa \varepsilon)+\kappa \varepsilon j_{n}^{\prime}(\kappa \varepsilon)}{h_{n}^{(1)}(\kappa \varepsilon)+\kappa \varepsilon h_{n}^{(1)^{\prime}}(\kappa \varepsilon)} \beta_{n, j}^{(1)}(\mathbf{x}) .
$$


Following the computations in the proof of (19), we deduce that

$$
\begin{gathered}
\boldsymbol{n}_{\varepsilon}(\mathbf{z}) \cdot \boldsymbol{E}_{\varepsilon}(\mathbf{z})=\zeta_{1}(\varepsilon) \sum_{j=-1}^{1} \beta_{1, j}^{(1)}(\mathbf{x}) \boldsymbol{\xi} \cdot N_{1, j}^{(1)}(\kappa, \varepsilon \boldsymbol{\xi})+O(\varepsilon)=3 \boldsymbol{\xi} \cdot \boldsymbol{E}^{\mathrm{inc}}(\mathbf{x})+O(\varepsilon), \\
\boldsymbol{n}_{\varepsilon}(\mathbf{z}) \times \operatorname{curl} \boldsymbol{E}_{\varepsilon}(\mathbf{z})=\gamma_{1}(\varepsilon) i \kappa \sum_{j=-1}^{1} \alpha_{1, j}^{(1)}(\mathbf{x}) \boldsymbol{\xi} \times N_{1, j}^{(1)}(\kappa, \varepsilon \boldsymbol{\xi})+O(\varepsilon)=\frac{3}{2} \boldsymbol{\xi} \times \operatorname{curl} \boldsymbol{E}^{\mathrm{inc}}(\mathbf{x})+O(\varepsilon) .
\end{gathered}
$$

The solution to the adjoint problem (19) can be written $\overline{\boldsymbol{W}}_{\varepsilon}=\overline{\boldsymbol{W}}_{\varepsilon}^{\text {scat }}+\overline{\boldsymbol{W}}_{\varepsilon}^{\text {inc }}$, where $\overline{\boldsymbol{W}}_{\varepsilon}^{\text {inc }}$ solves (29) with $\boldsymbol{E}_{\varepsilon}$ solving (1) for $\Omega=B_{\varepsilon}(\mathbf{x})$, and $\overline{\boldsymbol{W}}_{\varepsilon}^{\text {scat }}$ solves the following scattering problem:

$$
\begin{cases}\operatorname{curl} \mathbf{c u r l} \overline{\boldsymbol{W}}_{\varepsilon}^{\text {scat }}-\kappa^{2} \overline{\boldsymbol{W}}_{\varepsilon}^{\text {scat }}=\mathbf{0} & \text { in } \mathbb{R}^{3} \backslash \overline{B_{\varepsilon}(\mathbf{x})}, \\ \boldsymbol{n}_{\varepsilon} \times \overline{\boldsymbol{W}}_{\varepsilon}^{\text {scat }}=-\boldsymbol{n}_{\varepsilon} \times \overline{\boldsymbol{W}}_{\varepsilon}^{\text {inc }} & \text { on } \partial B_{\varepsilon}(\mathbf{x}), \\ \lim _{|\mathbf{z}| \rightarrow \infty}|\mathbf{z}|\left|\operatorname{curl} \overline{\boldsymbol{W}}_{\varepsilon}^{\text {scat }}(\mathbf{z}) \times \frac{\mathbf{z}}{|\mathbf{z}|}-i \kappa \overline{\boldsymbol{W}}_{\varepsilon}^{\text {scat }}(\mathbf{z})\right|=0 . & \end{cases}
$$

Then $\overline{\boldsymbol{W}}_{\varepsilon}^{\text {scat }}$ can be expressed in terms of the Mie series (34), and we immediately deduce that $\overline{\boldsymbol{W}}_{\varepsilon}$ has the same asymptotic behavior as $\boldsymbol{E}_{\varepsilon}$ when $\varepsilon \rightarrow 0$.

Proposition 9. Let $\boldsymbol{\xi} \in \mathbb{S}^{2}$. For the transmission problem, we have

$$
\begin{aligned}
& \lim _{\varepsilon \rightarrow 0} \boldsymbol{n}_{\varepsilon} \times \boldsymbol{E}_{\varepsilon}^{-}(\mathbf{x}+\varepsilon \boldsymbol{\xi})=\frac{3}{\frac{\beta_{i}^{2}}{\beta_{e}^{2}}+2} \boldsymbol{\xi} \times \boldsymbol{E}^{\mathrm{inc}}(\mathbf{x}), \\
& \lim _{\varepsilon \rightarrow 0} \boldsymbol{n}_{\varepsilon} \cdot \operatorname{curl} \boldsymbol{E}_{\varepsilon}^{-}(\mathbf{x}+\varepsilon \boldsymbol{\xi})=\frac{3}{1+2 \frac{\alpha_{i}}{\alpha_{e}}} \boldsymbol{\xi} \cdot \operatorname{curl} \boldsymbol{E}^{\mathrm{inc}}(\mathbf{x}), \\
& \lim _{\varepsilon \rightarrow 0} \boldsymbol{n}_{\varepsilon} \times \operatorname{curl} \boldsymbol{E}_{\varepsilon}^{-}(\mathbf{x}+\varepsilon \boldsymbol{\xi})=\frac{3}{1+2 \frac{\alpha_{i}}{\alpha_{e}}} \boldsymbol{\xi} \times \operatorname{curl} \boldsymbol{E}^{\mathrm{inc}}(\mathbf{x}), \\
& \lim _{\varepsilon \rightarrow 0} \boldsymbol{n}_{\varepsilon} \cdot \boldsymbol{E}_{\varepsilon}^{-}(\mathbf{x}+\varepsilon \boldsymbol{\xi})=\frac{3}{\frac{\beta_{i}^{2}}{\beta_{e}^{2}}+2} \boldsymbol{\xi} \cdot \boldsymbol{E}^{\mathrm{inc}}(\mathbf{x}),
\end{aligned}
$$

and $\overline{\boldsymbol{W}}_{\varepsilon}^{-}$has the same asymptotic behavior as $\boldsymbol{E}_{\varepsilon}^{-}$with $\boldsymbol{E}^{\text {inc }}$ replaced by $\frac{1}{\alpha_{e}} \overline{\boldsymbol{W}}_{0}^{\text {inc }}$.

Proof. Using the Mie series expansion, we find that

$$
\boldsymbol{E}_{\varepsilon}^{-}(\mathbf{z})=\sum_{n=1}^{\infty} \sum_{j=-n}^{n}\left[\alpha_{n, j}^{(2)}(\mathbf{x}, \varepsilon) M_{n, j}^{(1)}\left(\kappa_{i}, \mathbf{z}-\mathbf{x}\right)+\beta_{n, j}^{(2)}(\mathbf{x}, \varepsilon) N_{n, j}^{(1)}\left(\kappa_{i}, \mathbf{z}-\mathbf{x}\right)\right],
$$

where

$$
\alpha_{n, j}^{(2)}(\mathbf{x}, \varepsilon)=\frac{\kappa_{e} \varepsilon\left(j_{n}\left(\kappa_{e} \varepsilon\right) h_{n}^{(1)^{\prime}}\left(\kappa_{e} \varepsilon\right)-h_{n}^{(1)}\left(\kappa_{e} \varepsilon\right) j_{n}^{\prime}\left(\kappa_{e} \varepsilon\right)\right)}{j_{n}\left(\kappa_{i} \varepsilon\right)\left(h_{n}^{(1)}\left(\kappa_{e} \varepsilon\right)+\kappa_{e} \varepsilon h_{n}^{(1)^{\prime}}\left(\kappa_{e} \varepsilon\right)\right)-\frac{\alpha_{i}}{\alpha_{e}} h_{n}^{(1)}\left(\kappa_{e} \varepsilon\right)\left(j_{n}\left(\kappa_{i} \varepsilon\right)+\kappa_{i} \varepsilon j_{n}^{\prime}\left(\kappa_{i} \varepsilon\right)\right)} \alpha_{n, j}^{(1)}(\mathbf{x})
$$


and

$$
\beta_{n, j}^{(2)}(\mathbf{x}, \varepsilon)=\frac{\kappa_{i} \varepsilon\left(j_{n}\left(\kappa_{e} \varepsilon\right) h_{n}^{(1)^{\prime}}\left(\kappa_{e} \varepsilon\right)-h_{n}^{(1)}\left(\kappa_{e} \varepsilon\right) j_{n}^{\prime}\left(\kappa_{e} \varepsilon\right)\right)}{\frac{\beta_{i}^{2}}{\beta_{e}^{2}} j_{n}\left(\kappa_{i} \varepsilon\right)\left(h_{n}^{(1)}\left(\kappa_{e} \varepsilon\right)+\kappa_{e} \varepsilon h_{n}^{(1)^{\prime}}\left(\kappa_{e} \varepsilon\right)\right)-h_{n}^{(1)}\left(\kappa_{e} \varepsilon\right)\left(j_{n}\left(\kappa_{i} \varepsilon\right)+\kappa_{i} \varepsilon j_{n}^{\prime}\left(\kappa_{i} \varepsilon\right)\right)} \beta_{n, j}^{(1)}(\mathbf{x}) .
$$

If $\mathbf{z}=\mathbf{x}+\varepsilon \boldsymbol{\xi} \in \partial B_{\varepsilon}(\mathbf{x})$ with $\boldsymbol{\xi} \in \mathbb{S}^{2}$, then

$$
\begin{aligned}
\boldsymbol{n}_{\varepsilon}(\mathbf{z}) \times \boldsymbol{E}_{\varepsilon}^{-}(\mathbf{z}) & =\sum_{n=1}^{\infty} \sum_{j=-n}^{n}\left[\zeta_{n}^{1}(\varepsilon) \alpha_{n, j}^{(1)}(\mathbf{x}) \boldsymbol{\xi} \times M_{n, j}^{(1)}\left(\kappa_{e}, \varepsilon \boldsymbol{\xi}\right)+\gamma_{n}^{1}(\varepsilon) \beta_{n, j}^{(1)}(\mathbf{x}) \boldsymbol{\xi} \times N_{n, j}^{(1)}\left(\kappa_{e}, \varepsilon \boldsymbol{\xi}\right)\right], \\
\boldsymbol{n}_{\varepsilon}(\mathbf{z}) \cdot \operatorname{curl} \boldsymbol{E}_{\varepsilon}^{-}(\mathbf{z}) & =i \kappa_{e} \sum_{n=1}^{\infty} \sum_{j=-n}^{n}\left[\zeta_{n}^{1}(\varepsilon) \alpha_{n, j}^{(1)}(\mathbf{x}) \boldsymbol{\xi} \cdot N_{n, j}^{(1)}\left(\kappa_{e}, \varepsilon \boldsymbol{\xi}\right)\right], \\
\boldsymbol{n}_{\varepsilon}(\mathbf{z}) \times \operatorname{curl} \boldsymbol{E}_{\varepsilon}^{-}(\mathbf{z}) & =i \kappa_{e} \sum_{n=1}^{\infty} \sum_{j=-n}^{n}\left[\zeta_{n}^{2}(\varepsilon) \alpha_{n, j}^{(1)}(\mathbf{x}) \boldsymbol{\xi} \times N_{n, j}^{(1)}\left(\kappa_{e}, \varepsilon \boldsymbol{\xi}\right)-\gamma_{n}^{2}(\varepsilon) \beta_{n, j}^{(1)}(\mathbf{x}) \boldsymbol{\xi} \times M_{n, j}^{(1)}\left(\kappa_{e}, \varepsilon \boldsymbol{\xi}\right)\right], \\
\boldsymbol{n}_{\varepsilon}(\mathbf{z}) \cdot \boldsymbol{E}_{\varepsilon}^{-}(\mathbf{z}) & =\frac{\kappa_{e}^{2}}{\kappa_{i}^{2}} \sum_{n=1}^{\infty} \sum_{j=-n}^{n}\left[\gamma_{n}^{2}(\varepsilon) \beta_{n, j}^{(1)}(\mathbf{x}) \boldsymbol{\xi} \cdot N_{n, j}^{(1)}\left(\kappa_{e}, \varepsilon \boldsymbol{\xi}\right)\right],
\end{aligned}
$$

where

$$
\begin{aligned}
& \zeta_{n}^{1}(\varepsilon)= \frac{\kappa_{e} \varepsilon\left(j_{n}\left(\kappa_{e} \varepsilon\right) h_{n}^{(1)^{\prime}}\left(\kappa_{e} \varepsilon\right)-h_{n}^{(1)}\left(\kappa_{e} \varepsilon\right) j_{n}^{\prime}\left(\kappa_{e} \varepsilon\right)\right)}{j_{n}\left(\kappa_{i} \varepsilon\right)\left(h_{n}^{(1)}\left(\kappa_{e} \varepsilon\right)+\kappa_{e} \varepsilon h_{n}^{(1)^{\prime}}\left(\kappa_{e} \varepsilon\right)-\frac{\alpha_{i}}{\alpha_{e}} h_{n}^{(1)}\left(\kappa_{e} \varepsilon\right)\left(j_{n}\left(\kappa_{i} \varepsilon\right)+\kappa_{i} \varepsilon j_{n}^{\prime}\left(\kappa_{i} \varepsilon\right)\right)\right.} \cdot \frac{j_{n}\left(\kappa_{i} \varepsilon\right)}{j_{n}\left(\kappa_{e} \varepsilon\right)} \\
& \underset{\varepsilon \rightarrow 0}{\approx} \frac{2 n+1}{n+\frac{\alpha_{i}}{\alpha_{e}}(n+1)}, \\
& \gamma_{n}^{1}(\varepsilon)= \frac{\kappa_{i} \varepsilon\left(j_{n}\left(\kappa_{e} \varepsilon\right) h_{n}^{(1)^{\prime}}\left(\kappa_{e} \varepsilon\right)-h_{n}^{(1)}\left(\kappa_{e} \varepsilon\right) j_{n}^{\prime}\left(\kappa_{e} \varepsilon\right)\right)}{\frac{\beta_{i}^{2}}{\beta_{e}^{2}} j_{n}\left(\kappa_{i} \varepsilon\right)\left(h_{n}^{(1)}\left(\kappa_{e} \varepsilon\right)+\kappa_{e} \varepsilon h_{n}^{(1)^{\prime}}\left(\kappa_{e} \varepsilon\right)\right)-h_{n}^{(1)}\left(\kappa_{e} \varepsilon\right)\left(j_{n}\left(\kappa_{i} \varepsilon\right)+\kappa_{i} \varepsilon j_{n}^{\prime}\left(\kappa_{i} \varepsilon\right)\right)} \\
& \cdot \frac{j_{n}\left(\kappa_{i} \varepsilon\right)+\kappa_{i} \varepsilon j_{n}^{\prime}\left(\kappa_{i} \varepsilon\right)}{j_{n}\left(\kappa_{e} \varepsilon\right)+\kappa_{e} \varepsilon j_{n}^{\prime}\left(\kappa_{e} \varepsilon\right)} \cdot \frac{\kappa_{e}}{\kappa_{i}} \\
& \underset{\varepsilon \rightarrow 0}{\approx} \frac{2 n+1}{\frac{\beta_{i}^{2}}{\beta_{e}^{2}} n+(n+1)}, \\
& \zeta_{n}^{2}(\varepsilon)= \frac{\kappa_{e} \varepsilon\left(j_{n}\left(\kappa_{e} \varepsilon\right) h_{n}^{(1)^{\prime}}\left(\kappa_{e} \varepsilon\right)-h_{n}^{(1)}\left(\kappa_{e} \varepsilon\right) j_{n}^{\prime}\left(\kappa_{e} \varepsilon\right)\right)}{j_{n}\left(\kappa_{i} \varepsilon\right)\left(h_{n}^{(1)}\left(\kappa_{e} \varepsilon\right)+\kappa_{e} \varepsilon h_{n}^{(1)^{\prime}}\left(\kappa_{e} \varepsilon\right)\right)-\frac{\alpha_{i}}{\alpha_{e}} h_{n}^{(1)}\left(\kappa_{e} \varepsilon\right)\left(j_{n}\left(\kappa_{i} \varepsilon\right)+\kappa_{i} \varepsilon j_{n}^{\prime}\left(\kappa_{i} \varepsilon\right)\right)} \cdot \frac{j_{n}\left(\kappa_{i} \varepsilon\right)+\kappa_{i} \varepsilon j_{n}^{\prime}\left(\kappa_{i} \varepsilon\right)}{j_{n}\left(\kappa_{e} \varepsilon\right)+\kappa_{e} \varepsilon j_{n}^{\prime}\left(\kappa_{e} \varepsilon\right)} \\
& \underset{\varepsilon \rightarrow 0}{\approx} \frac{2 n+1}{n+\frac{\alpha_{i}}{\alpha_{e}}(n+1)}, \\
& \gamma_{n}^{2}(\varepsilon)= \frac{\kappa_{i} \varepsilon\left(j_{n}\left(\kappa_{e} \varepsilon\right) h_{n}^{(1)^{\prime}}\left(\kappa_{e} \varepsilon\right)-h_{n}^{(1)}\left(\kappa_{e} \varepsilon\right) j_{n}^{\prime}\left(\kappa_{e} \varepsilon\right)\right)}{\frac{\beta_{i}^{2}}{\beta_{e}^{2}} j_{n}\left(\kappa_{i} \varepsilon\right)\left(h_{n}^{(1)}\left(\kappa_{e} \varepsilon\right)+\kappa_{e} \varepsilon h_{n}^{(1)^{\prime}}\left(\kappa_{e} \varepsilon\right)\right)-h_{n}^{(1)}\left(\kappa_{e} \varepsilon\right)\left(j_{n}\left(\kappa_{i} \varepsilon\right)+\kappa_{i} \varepsilon j_{n}^{\prime}\left(\kappa_{i} \varepsilon\right)\right)} \cdot \frac{j_{n}\left(\kappa_{i} \varepsilon\right)}{j_{n}\left(\kappa_{e} \varepsilon\right)} \cdot \frac{\kappa_{i}}{\kappa_{e}} \\
& \underset{\varepsilon \rightarrow 0}{\approx} \frac{2 n+1}{\frac{\beta_{i}^{2}}{\beta_{e}^{2}} n+(n+1)} \cdot \frac{\kappa_{i}^{2}}{\kappa_{e}^{2}} \cdot
\end{aligned}
$$


The solution to the adjoint problem (22) can be written $\overline{\boldsymbol{W}}_{\varepsilon}=\overline{\boldsymbol{W}}_{\varepsilon}^{\text {scat }}+\frac{1}{\alpha_{e}} \overline{\boldsymbol{W}}_{\varepsilon}^{\mathrm{inc}}$, where $\overline{\boldsymbol{W}}_{\varepsilon}^{\text {inc }}$ solves (29) with $\boldsymbol{E}_{\varepsilon}$ solving (3) with $\Omega=B_{\varepsilon}(\mathbf{x})$, and $\overline{\boldsymbol{W}}_{\varepsilon}^{\text {scat }}$ solves the following scattering problem:

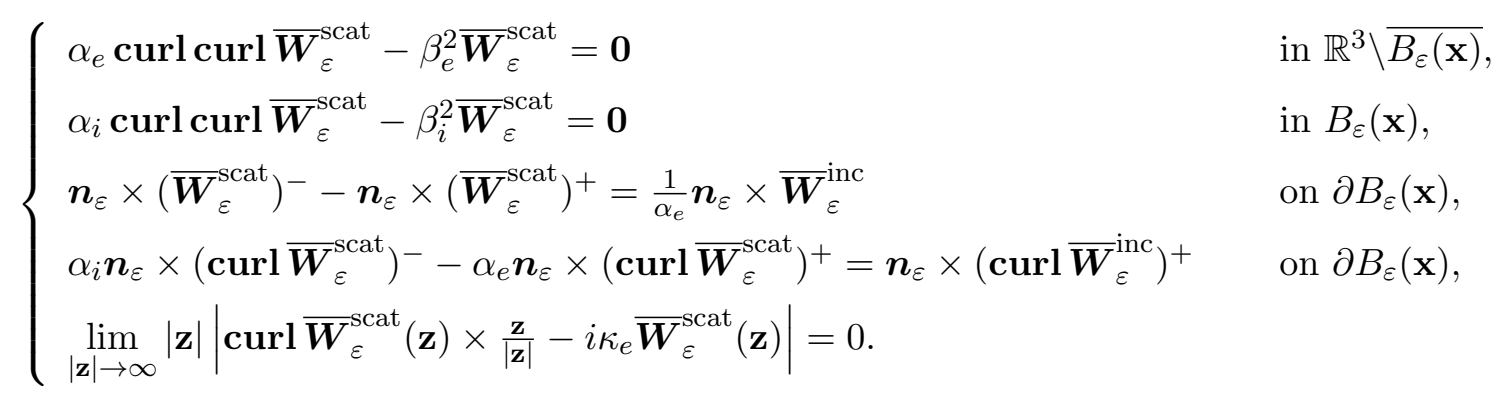

Then $\overline{\boldsymbol{W}}_{\varepsilon}^{\text {scat }}$ can be expressed in terms of the Mie series (35), from which we easily deduce the asymptotic behavior of $\overline{\boldsymbol{W}}_{\varepsilon}$ when $\varepsilon \rightarrow 0$.

Theorem 10. Depending on the selected boundary condition, the topological derivative at $\mathbf{x} \in \mathbb{R}^{3}$ of the cost function $J$ defined in (9) is given by:

(i) Perfect conductors (Dirichlet boundary condition),

$$
\mathcal{D}_{T}(\mathbf{x})=\frac{3}{2} R e\left[\sum_{r=1}^{N_{\mathrm{inc}}}\left(\operatorname{curl} \boldsymbol{E}_{r}^{\mathrm{inc}}(\mathbf{x}) \cdot \operatorname{curl} \overline{\boldsymbol{W}}_{r, 0}^{\mathrm{inc}}(\mathbf{x})-2 \kappa^{2} \boldsymbol{E}_{r}^{\mathrm{inc}}(\mathbf{x}) \cdot \overline{\boldsymbol{W}}_{r, 0}^{\mathrm{inc}}(\mathbf{x})\right)\right] .
$$

(ii) Electromagnetic cavities (Neumann condition),

$$
\mathcal{D}_{T}(\mathbf{x})=\frac{3}{2} \operatorname{Re}\left[\sum_{r=1}^{N_{\text {inc }}}\left(\kappa^{2} \boldsymbol{E}_{r}^{\mathrm{inc}}(\mathbf{x}) \cdot \overline{\boldsymbol{W}}_{r, 0}^{\mathrm{inc}}(\mathbf{x})-2 \operatorname{curl} \boldsymbol{E}_{r}^{\mathrm{inc}}(\mathbf{x}) \cdot \operatorname{curl} \overline{\boldsymbol{W}}_{r, 0}^{\mathrm{inc}}(\mathbf{x})\right)\right] .
$$

(iii) Absorbing obstacles (impedance boundary condition with $\lambda \neq 0$ ),

$$
\mathcal{D}_{T}(\mathbf{x})=-3 R e\left[\sum _ { r = 1 } ^ { N _ { \mathrm { inc } } } \left(\kappa^{2} \boldsymbol{E}_{r}^{\mathrm{inc}}(\mathbf{x}) \cdot \overline{\boldsymbol{W}}_{r, 0}^{\mathrm{inc}}(\mathbf{x})+\operatorname{curl} \boldsymbol{E}_{r}^{\mathrm{inc}}(\mathbf{x}) \cdot \operatorname{curl} \overline{\left.\left.\boldsymbol{W}_{r, 0}^{\mathrm{inc}}(\mathbf{x})\right)\right] .} .\right.\right.
$$

(iv) Dielectric inclusions (transmission conditions),

$$
\mathcal{D}_{T}(\mathbf{x})=3 R e\left[\sum_{r=1}^{N_{\text {inc }}}\left(\frac{\beta_{e}^{2}\left(\beta_{e}^{2}-\beta_{i}^{2}\right)}{\alpha_{e}\left(\beta_{i}^{2}+2 \beta_{e}^{2}\right)} \boldsymbol{E}_{r}^{\mathrm{inc}}(\mathbf{x}) \cdot \overline{\boldsymbol{W}}_{r, 0}^{\mathrm{inc}}(\mathbf{x})-\frac{\alpha_{e}-\alpha_{i}}{\alpha_{e}+2 \alpha_{i}} \operatorname{curl} \boldsymbol{E}_{r}^{\mathrm{inc}}(\mathbf{x}) \cdot \operatorname{curl} \overline{\boldsymbol{W}}_{r, 0}^{\mathrm{inc}}(\mathbf{x})\right)\right],
$$

where

$$
\overline{\boldsymbol{W}}_{r, 0}^{\text {inc }}(\mathbf{z})=\frac{1}{\kappa^{2}} \operatorname{curl}^{\operatorname{curl}^{\mathbf{z}}}\left\{\int_{\Gamma_{\text {meas }}} G\left(\kappa, \mathbf{z}-\mathbf{y}_{\text {obs }}\right)\left(\overline{\boldsymbol{E}_{\text {meas }, r}\left(\mathbf{y}_{\text {obs }}\right)-\boldsymbol{E}_{r}^{\text {inc }}\left(\mathbf{y}_{\text {obs }}\right)}\right) d \mu\left(\mathbf{y}_{\text {obs }}\right)\right\} .
$$

Proof. We use the following formulae:

$\int_{\partial B_{\varepsilon}(\mathbf{x})}(\boldsymbol{\xi}(\mathbf{y}) \cdot \mathbf{a})(\boldsymbol{\xi}(\mathbf{y}) \cdot \mathbf{b}) d \sigma(\mathbf{y})=\frac{4}{3} \pi \varepsilon^{2} \mathbf{a} \cdot \mathbf{b}, \quad \int_{\partial B_{\varepsilon}(\mathbf{x})}(\boldsymbol{\xi}(\mathbf{y}) \times \mathbf{a}) \cdot(\boldsymbol{\xi}(\mathbf{y}) \times \mathbf{b}) d \sigma(\mathbf{y})=\frac{8}{3} \pi \varepsilon^{2} \mathbf{a} \cdot \mathbf{b}$, where $\boldsymbol{\xi}(\mathbf{y})=\frac{\mathbf{y}-\mathbf{x}}{\varepsilon}$ and $\mathbf{a}, \mathbf{b} \in \mathbb{R}^{3}$. 
We end this section by extending the previous results to the case of far-field data. We denote by $\mathcal{D}_{T}^{\infty}(\mathbf{x})$ the topological derivative of the cost function with far-field measurements. Setting $\mathbf{y}_{\text {obs }}=R \widehat{\mathbf{y}}_{\text {obs }}$, then we have either

$$
\begin{aligned}
J\left(\mathbb{R}^{3} \backslash \Omega\right) & =\sum_{r=1}^{N_{\text {inc }}} \frac{1}{2} \int_{\mathbb{S}^{2}}\left|\boldsymbol{E}_{r}^{\infty}\left(\widehat{\mathbf{y}}_{\text {obs }}\right)-\boldsymbol{E}_{\text {meas }, r}^{\infty}\left(\widehat{\mathbf{y}}_{\text {obs }}\right)\right|^{2} d \mu\left(\widehat{\mathbf{y}}_{\text {obs }}\right) \\
& =\lim _{R \rightarrow \infty} \sum_{r=1}^{N_{\text {inc }}} \frac{1}{2} \int_{\mathbb{S}^{2}} R^{2}\left|\boldsymbol{E}_{r}\left(R \widehat{\mathbf{y}}_{\text {obs }}\right)-\boldsymbol{E}_{\text {meas }, r}\left(R \widehat{\mathbf{y}}_{\text {obs }}\right)\right|^{2} d \mu\left(\widehat{\mathbf{y}}_{\text {obs }}\right) \\
& =\lim _{R \rightarrow \infty} \sum_{r=1}^{N_{\text {inc }}} \frac{1}{2} \int_{\partial B_{R}(\mathbf{0})}\left|\boldsymbol{E}_{r}\left(\mathbf{y}_{\text {obs }}\right)-\boldsymbol{E}_{\text {meas }, r}\left(\mathbf{y}_{\text {obs }}\right)\right|^{2} d \mu\left(\mathbf{y}_{\text {obs }}\right)
\end{aligned}
$$

or, with Dirac measures,

$$
\begin{aligned}
J\left(\mathbb{R}^{3} \backslash \Omega\right) & =\sum_{r=1}^{N_{\text {inc }}} \frac{1}{2} \sum_{s=1}^{N_{\text {obs }}}\left|\boldsymbol{E}_{r}^{\infty}\left(\widehat{\mathbf{y}}_{\text {obs }}^{s}\right)-\boldsymbol{E}_{\text {meas }, r}^{\infty}\left(\widehat{\mathbf{y}}_{\text {obs }}^{s}\right)\right|^{2} \\
& =\lim _{R \rightarrow \infty} \sum_{r=1}^{N_{\text {inc }}} \frac{1}{2} \sum_{s=1}^{N_{\text {obs }}} R^{2}\left|\boldsymbol{E}_{r}\left(\mathbf{y}_{\text {obs }}^{s}\right)-\boldsymbol{E}_{\text {meas }, r}\left(\mathbf{y}_{\text {obs }}^{s}\right)\right|^{2} .
\end{aligned}
$$

It follows that $\mathcal{D}_{T}^{\infty}$ can be obtained as a limit of $\mathcal{D}_{T}$ with $\Gamma_{\text {meas }}=\partial B_{R}(\mathbf{0})$ when $R \rightarrow \infty$. Thanks to the radiation condition, we have

$$
\lim _{\left|\mathbf{y}_{\text {obs }}\right| \rightarrow \infty}\left|\mathbf{y}_{\text {obs }}\right|^{2} G\left(\kappa, \mathbf{z}-\mathbf{y}_{\text {obs }}\right)\left(\overline{\boldsymbol{E}_{\text {meas }}\left(\mathbf{y}_{\text {obs }}\right)-\boldsymbol{E}^{\text {inc }}\left(\mathbf{y}_{\text {obs }}\right)}\right)=\frac{1}{4 \pi} e^{-i \kappa \mathbf{z} \cdot \widehat{\mathbf{y}}_{\text {obs }}} \overline{\boldsymbol{E}_{\text {meas }}^{\infty}\left(\widehat{\mathbf{y}}_{\text {obs }}\right)},
$$

uniformly in all directions $\widehat{\mathbf{y}}_{\text {obs }}=R^{-1} \mathbf{y}_{\text {obs }} \in \mathbb{S}^{2}$. We deduce the following theorem.

Theorem 11. Depending on the selected boundary condition, the topological derivative at $\mathbf{x} \in \mathbb{R}^{3}$ of the cost function $J$ defined in (10) with far-field data is given by:

(i) Perfect conductors (Dirichlet problem),

$$
\mathcal{D}_{T}^{\infty}(\mathbf{x})=\frac{3}{2} R e\left[\sum_{r=1}^{N_{\mathrm{inc}}}\left(\operatorname{curl} \boldsymbol{E}_{r}^{\mathrm{inc}}(\mathbf{x}) \cdot \operatorname{curl} \overline{\boldsymbol{W}}_{r, 0}^{\mathrm{inc}}(\mathbf{x})-2 \kappa^{2} \boldsymbol{E}_{r}^{\mathrm{inc}}(\mathbf{x}) \cdot \overline{\boldsymbol{W}}_{r, 0}^{\mathrm{inc}}(\mathbf{x})\right)\right] .
$$

(ii) Electromagnetic cavities (Neumann problem),

$$
\mathcal{D}_{T}^{\infty}(\mathbf{x})=\frac{3}{2} R e\left[\sum_{r=1}^{N_{\mathrm{inc}}}\left(\kappa^{2} \boldsymbol{E}_{r}^{\mathrm{inc}}(\mathbf{x}) \cdot \overline{\boldsymbol{W}}_{r, 0}^{\mathrm{inc}}(\mathbf{x})-2 \operatorname{curl} \boldsymbol{E}_{r}^{\mathrm{inc}}(\mathbf{x}) \cdot \operatorname{curl} \overline{\boldsymbol{W}}_{r, 0}^{\mathrm{inc}}(\mathbf{x})\right)\right] .
$$

(iii) Absorbing obstacles (impedance problem with $\lambda \neq 0$ ),

$$
\mathcal{D}_{T}^{\infty}(\mathbf{x})=-3 \operatorname{Re}\left[\sum_{r=1}^{N_{\text {inc }}}\left(\kappa^{2} \boldsymbol{E}_{r}^{\mathrm{inc}}(\mathbf{x}) \cdot \overline{\boldsymbol{W}}_{r, 0}^{\mathrm{inc}}(\mathbf{x})+\operatorname{curl} \boldsymbol{E}_{r}^{\mathrm{inc}}(\mathbf{x}) \cdot \operatorname{curl} \overline{\boldsymbol{W}}_{r, 0}^{\mathrm{inc}}(\mathbf{x})\right)\right] .
$$


(iv) Dielectric inclusions (transmission problem),

$$
\mathcal{D}_{T}^{\infty}(\mathbf{x})=3 R e\left[\sum_{r=1}^{N_{\text {inc }}}\left(\frac{\beta_{e}^{2}\left(\beta_{e}^{2}-\beta_{i}^{2}\right)}{\alpha_{e}\left(\beta_{i}^{2}+2 \beta_{e}^{2}\right)} \boldsymbol{E}_{r}^{\mathrm{inc}}(\mathbf{x}) \cdot \overline{\boldsymbol{W}}_{r, 0}^{\mathrm{inc}}(\mathbf{x})-\frac{\alpha_{e}-\alpha_{i}}{\alpha_{e}+2 \alpha_{i}} \operatorname{curl} \boldsymbol{E}_{r}^{\mathrm{inc}}(\mathbf{x}) \cdot \operatorname{curl} \overline{\boldsymbol{W}}_{r, 0}^{\mathrm{inc}}(\mathbf{x})\right)\right],
$$

where

$$
\overline{\boldsymbol{W}}_{r, 0}^{\mathrm{inc}}(\mathbf{z})=\frac{1}{\kappa^{2}} \operatorname{curl}_{\mathbf{c u r l}}^{\mathbf{z}}\left\{\sum_{s=1}^{N_{\mathrm{obs}}} \frac{1}{4 \pi} e^{-i \kappa \mathbf{z} \cdot \widehat{\mathbf{y}}_{\mathrm{obs}}^{s} \overline{\boldsymbol{E}_{\text {meas }, r}^{\infty}\left(\widehat{\mathbf{y}}_{\mathrm{obs}}^{s}\right)}}\right\} .
$$

Remark 2. The topological derivative formula (iv) for the detection of dielectric inclusions coincides with one previously obtained in [40, Corollary 1] for near-field data. In this case, the stability of the resolution method with respect to measurement or medium noises is analyzed in [52]. We also point out that the formulae for the perfect conductors, electromagnetic cavities, and absorbing obstacles cases can be formally deduced from (iv). Passing to the limits $\beta_{i} \rightarrow \infty$ and $\alpha_{i} \rightarrow \infty$ gives (i), $\beta_{i} \rightarrow 0$ and $\alpha_{i} \rightarrow 0$ gives (ii), and $\beta_{i} \rightarrow \infty, \alpha_{i} \rightarrow 0$ gives (iii). However, the formula for the Neumann problem cannot be obtained by passing to the limit $\lambda \rightarrow 0$ in the formula (iii) for absorbing obstacles.

4. Numerical examples. In this section, we illustrate the applicability of the one step method by showing various numerical examples in three dimensions for the Neumann boundary condition and near-field data from full aperture angles (incident directions spanning the unit sphere) at full aperture observation points. Other boundary conditions, limited aperture angles and limited aperture observations, far-field data, and noisy data are dealt with in the supplementary material file M111385_01.pdf [local/web 4.66MB]. All our experiments are realized using the MATLAB programming language.

4.1. Synthetic data. To produce synthetic data we solve the direct scattering problems using boundary integral equation methods and the fast spectral algorithm developed for single and multiple scattering problems in [19, 20, 21, 22]. For the Dirichlet and Neumann problems we use the modified-combined field integral equation method described in [34]. For the Leontovitch impedance problem, we use the combined-field formulation described in [5]. For the dielectric scattering problem, we use the so-called Muller's system, also described in [35, 39].

The spectral algorithm applies to boundaries $\partial \Omega$ each of whose simply connected components $\partial \Omega_{j}, j=1, \ldots, N$, can be described explicitly in a spherical coordinates system. The original obstacles $\Omega_{j}, j=1, \ldots, N$, are defined by a center $\boldsymbol{c}_{j} \in \mathbb{R}^{3}$ and a spherical boundary parametrization such that $\partial \Omega_{j}=\boldsymbol{c}_{j}+\boldsymbol{q}_{j}\left(\mathbb{S}^{2}\right)$ with $\boldsymbol{q}_{j} \in \mathscr{C}^{1}\left(\mathbb{S}^{2}, \mathbb{R}^{3}\right)$. The boundary integral equations are transported on the unit sphere. Then the full system is numerically solved to obtain the scattered field using a Galerkin scheme where the solution space is spanned by the complete family of tangential vector spherical harmonics of order lower than $n_{\text {sol }} \in \mathbb{N}$ with $n_{\text {sol }} \geq 5$ and arbitrarily chosen.

In all the experiments in this section a pseudorandom $1 \%$ relative noise is added to the scattered fields, generated by using the function randn of MATLAB. Numerical experiments dealing with higher noise levels are provided in the supplementary material file M111385_01.pdf [local/web $4.66 \mathrm{MB}]$. 
4.2. Reconstructed defects. We assume that the obstacles are lying inside a cubic region $[-d, d]^{3}$ with $d>0$ (in all our experiments $d=3$ ). We discretize this region by an equidistributed sample set $\mathcal{R}_{N_{d}}$ of $\left(N_{d}+1\right)^{3}$ points defined by $\mathbf{x}=\left(x_{1}^{k_{1}}, x_{2}^{k_{2}}, x_{3}^{k_{3}}\right)$, where $x_{i}^{k_{i}}=-d+k_{i} \frac{2 d}{N_{d}}$ for $i=1,2,3$ and $k_{i}=0, \ldots, N_{d}$. We compute the topological derivative $\mathcal{D}_{T}(\mathbf{x})$ for any $\mathbf{x} \in \mathcal{R}_{N_{d}}$ and define the following point cloud:

$$
\Omega_{\mathrm{app}}=\left\{\mathbf{x} \in \mathcal{R}_{N_{d}} ; \mathcal{D}_{T}(\mathbf{x})<\left(1-C_{0}\right) \min _{\mathbf{y} \in \mathcal{R}_{N_{d}}} \mathcal{D}_{T}(\mathbf{y})\right\}
$$

where $C_{0}>0$ is arbitrarily chosen. Our objective is then to find the number of simple connected components of $\Omega_{\mathrm{app}}$. To do so, the condition $\mathcal{D}_{T}(\mathbf{x})<\left(1-C_{0}\right) \min _{\mathbf{y} \in \mathcal{R}_{N_{d}}} \mathcal{D}_{T}(\mathbf{y})$ is collected in a 3D binary matrix $\mathrm{M}$ whose size is $\left(N_{d}+1\right)^{3}$. The MATLAB command

$$
\mathrm{CC}=\mathrm{bwconn} \operatorname{comp}(\mathrm{M}) \text {; }
$$

provides the connected point cloud of the domain $\Omega_{\mathrm{app}}$. Let $N_{\text {rec }}$ be the number of detected obstacles. To visualize the domain $\Omega_{\text {app }}$ we can use, for $j=1, \ldots, N_{r e c}$, the following MATLAB commands:

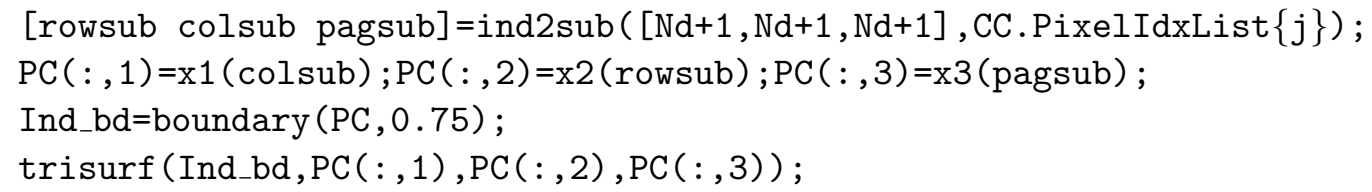

In order to both visualize the domain $\Omega_{\text {app }}$ and to define $\Omega_{\text {app }}$ in a suitable way for future applications for iterative algorithms that require an initial guess, we explain now how to postprocess the points that define $\partial \Omega_{\text {app }}$ to approximate each simple connected component $\Omega_{j}^{\text {app }}$ by a star-shaped defect, given by a boundary parametrization of the form $\boldsymbol{c}_{j}^{\text {app }}+\boldsymbol{q}_{j}^{\text {app }}\left(\mathbb{S}^{2}\right)$. This is very useful in dealing with iterative methods coupled to the fast spectral direct solver $[19,20,21,22]$ considered in this paper, and for any other direct solver requiring an explicit parametrization of the boundary, as, e.g., some boundary integral discretizations. However, it is not mandatory to approximate $\Omega_{\text {app }}$ by star-shaped objects. In particular, for approaches related to finite element discretizations, other approximations could be of interest, such as the one proposed in [10], based on the open access mesher DistMesh, described in [47]. For $j=1, \ldots, N_{\text {rec }}$, the following MATLAB commands provide the approximate center $\boldsymbol{c}_{j}^{\text {app }}$ and some points located on the approximate boundary $\boldsymbol{q}_{j}^{\text {app }}\left(\mathbb{S}^{2}\right)$ :

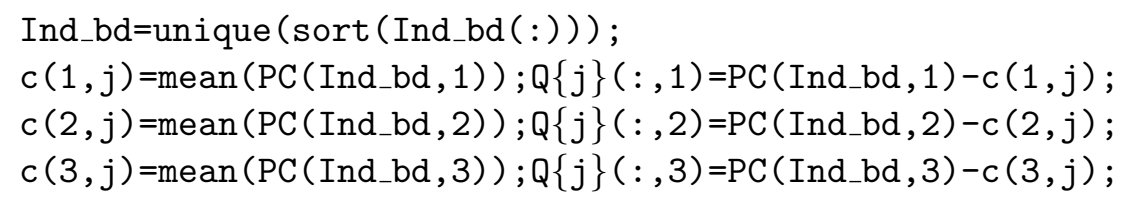

We then look for a radial parametrization of $\boldsymbol{q}_{j}^{\text {app }}\left(\mathbb{S}^{2}\right)$, which means that we set $\boldsymbol{q}_{j}^{\text {app }}(\hat{\mathbf{x}})=$ $\hat{\mathbf{x}} r_{j}^{\text {app }}(\hat{\mathbf{x}})$ and

$$
r_{j}^{\mathrm{app}}=\sum_{\ell=0}^{n_{\text {star }}} \sum_{m=-\ell}^{\ell} \alpha_{\ell}^{m} Y_{\ell}^{m},
$$


(a)

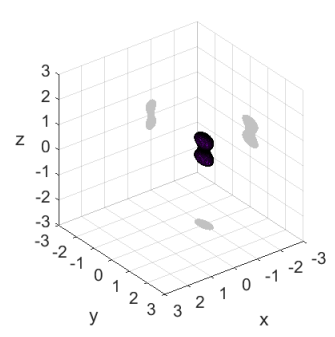

(b)

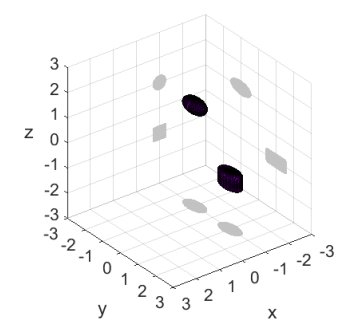

(c)

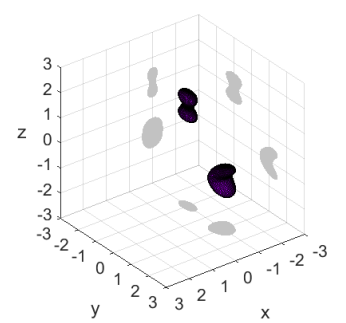

(d)

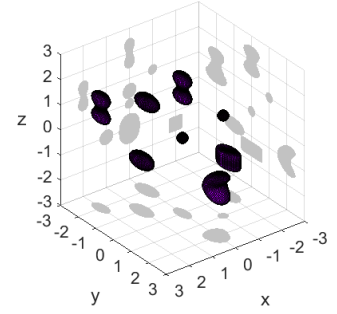

Figure 1. True geometries considered for the examples: (a) a single peanut-like defect; (b) two convex domains, an ellipsoid and a rounded-cylinder; (c) two nonconvex defects with beam-like and peanut-like shapes; (d) a configuration with eight defects with different shapes and sizes.

where $Y_{\ell}^{m}$ for $\ell \geq 0$ and $|m| \leq \ell$ are the scalar spherical harmonics defined in [35, Appendix A]. To compute the Fourier coefficients $\alpha_{\ell}^{m}$, we first compute the function $r_{j}^{\text {app }}$ at the Gauss quadrature points [19, eq. (2.43)], as follows:

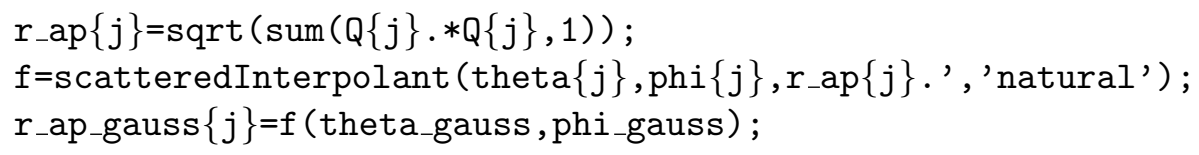

Then we use the exact quadrature rule [19, eq. (2.42)] for spherical harmonics to evaluate

$$
\alpha_{\ell}^{m}=\int_{\mathbb{S}^{2}} r_{j}^{\mathrm{app}}(\hat{\mathbf{x}}) \overline{Y_{\ell}^{m}(\hat{\mathbf{x}})} d s(\hat{\mathbf{x}})
$$

4.3. Numerical experiments for the Neumann problem. Let us consider the Neumann problem, namely, problem (2) with $\lambda=0$. The true domain for the first experiment is the peanut-like object represented in Figure 1(a). We generate 12 incident waves of the form $\boldsymbol{E}_{r}^{\text {inc }}(\mathbf{x})=\boldsymbol{p}_{r} e^{i \kappa \mathbf{x} \cdot \boldsymbol{d}_{r}}$, for $\kappa=3$, where the incident directions $\mathbf{d}_{r}$ are defined by the 12 vertices of a regular icosahedron, and the corresponding polarization direction is either $\mathbf{p}_{r}=(1,0,0),(0,1,0)$, or $(0,0,1)$ (the one that is orthogonal to the corresponding $\left.\mathbf{d}_{r}\right)$. Measurements are taken on 128 points located on the sphere centered at the origin and with radius 6. We compute the TD at the cubic region $[-3,3]^{3}$. We show in the first row of Figure 2 the values of the TD at three planes that intersect at the center of the true object: $x=-1$, $y=0$, and $z=0$. The section of the true object by each plane is represented by a black line. In the second row, the TD is represented at several planes that are parallel to these (see also the associated movie M111385_02.avi [local/web 2.04MB], which shows the values of the TD at a series of planes). We observe that the most pronounced negative values of the TD (blue colors in the plots) are attained inside the true scatterer. The reconstructed objects obtained by following the procedure described in section 4.2 to find star-shaped approximations when taking $C_{0}=0.3,0.45$, and 0.6 in the definition (36) are shown in the third row of Figure 2 . The method localizes the position sharply with a good approximation of its volume (which obviously depends on the threshold $C_{0}$ ). We observe that the reconstructed defect is not too sensitive to the threshold $C_{0}$, which means that the TD is quite steep near the boundary of the reconstructed defect. Nevertheless, the shape is not well recovered, and the object seems 

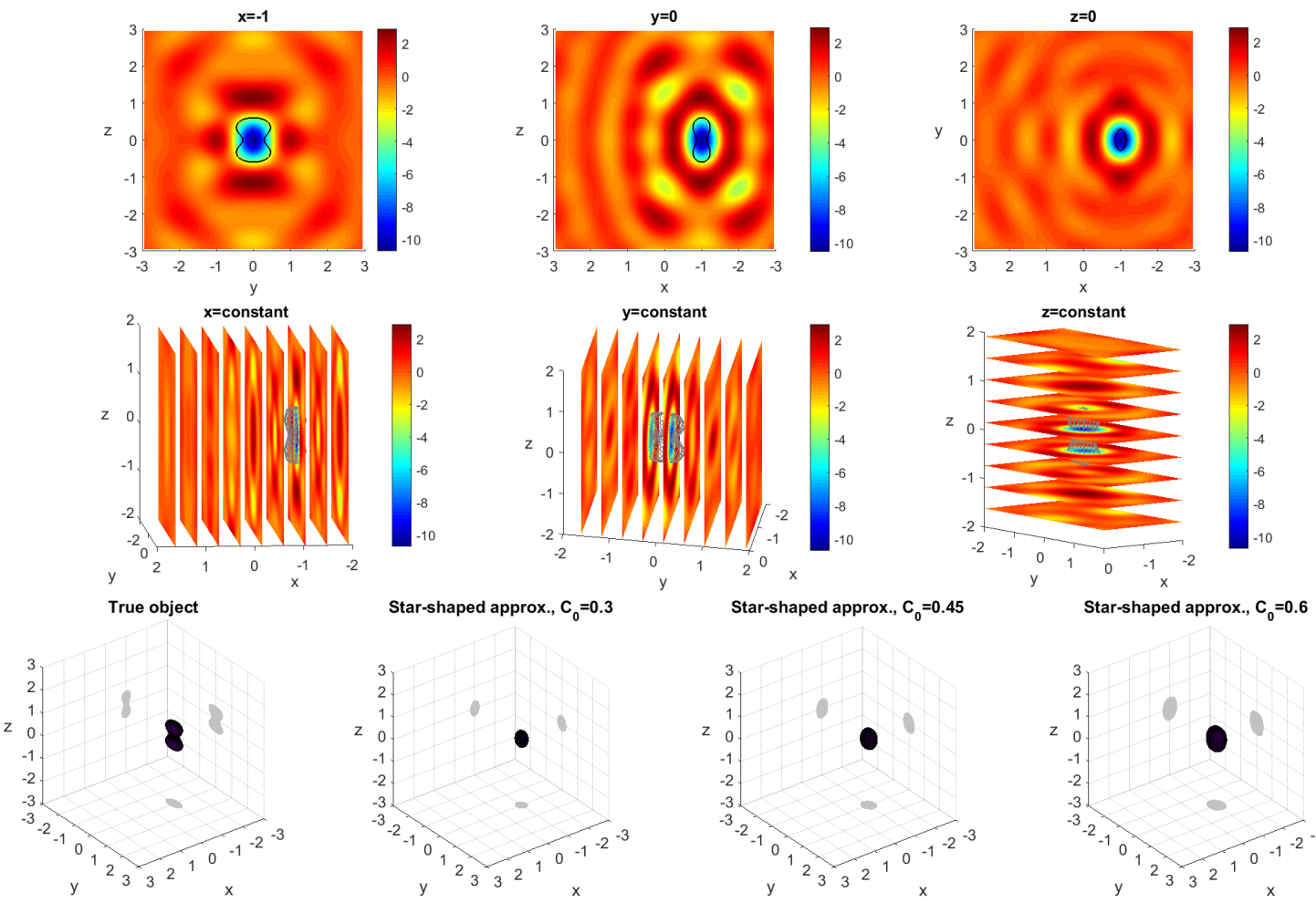

Figure 2. First and second rows: Values of the TD for the Neumann problem with $\kappa=3$ at several planes parallel to $x=0, y=0$, and $z=0$, when 12 incident waves and 128 measurement points are considered. Third row: True and reconstructed defect when taking $C_{0}=0.3,0.45$, and 0.6 in the definition (36).

to be an ellipsoid instead of a peanut-like object. Increasing the wavenumber to $\kappa=4$ (see Figure 3 and the associated movie M111385_03.avi [local/web 2.01MB]), we obtain a sharper approximation of the true shape, although for $C_{0}=0.3$ it seems that we have two closely located ellipsoids instead of just one peanut-like obstacle. For the value $C_{0}=0.6$, the fidelity of the reconstruction is impressive. The wavenumber $\kappa=5$ provides a reconstruction with two defects, but the overall reconstruction is also satisfactory, as can be observed in Figure 4 and in the associated movie M111385_04.avi [local/web 2.06MB]. It is also noticeable that the higher the wavenumber is, the more oscillatory the TD is.

Let us now consider the geometrical configuration with two defects shown in Figure 1(b), with an ellipsoid and a rounded cylinder (that is, a cylinder with smooth edges). The objective is twofold: first to test the performance of the method when two defects are present, and second, to explore the ability of the method to distinguish between two shapes that are similar. We keep the original base parameters: 12 incident directions defined by the vertices of the icosahedron, and 128 measurement points on the sphere centered at the origin and with radius 6 . We compare in Figure 5 the reconstructions for $C_{0}=0.6$ when $\kappa=3$ (first row) and $\kappa=5$ (second row). For the sake of brevity, we only show the TD at the plane $x=-1$ and at some planes of the form $z=z_{0}$. The associated movies M111385_05.avi [local/web 2.06MB] and M111385_06.avi [local/web 2.13MB] display the TD at different planes for $\kappa=3$ 

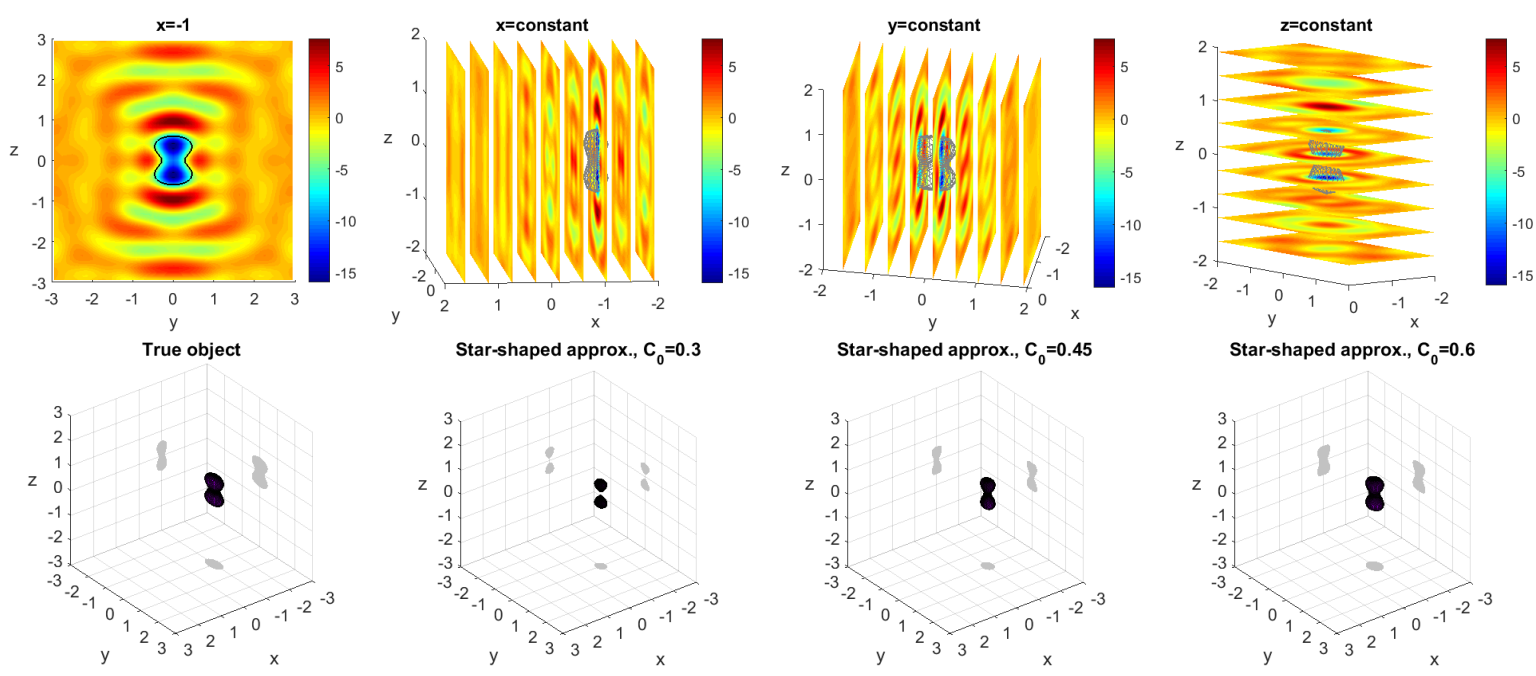

Figure 3. Counterpart of Figure 2 for $\kappa=4$.
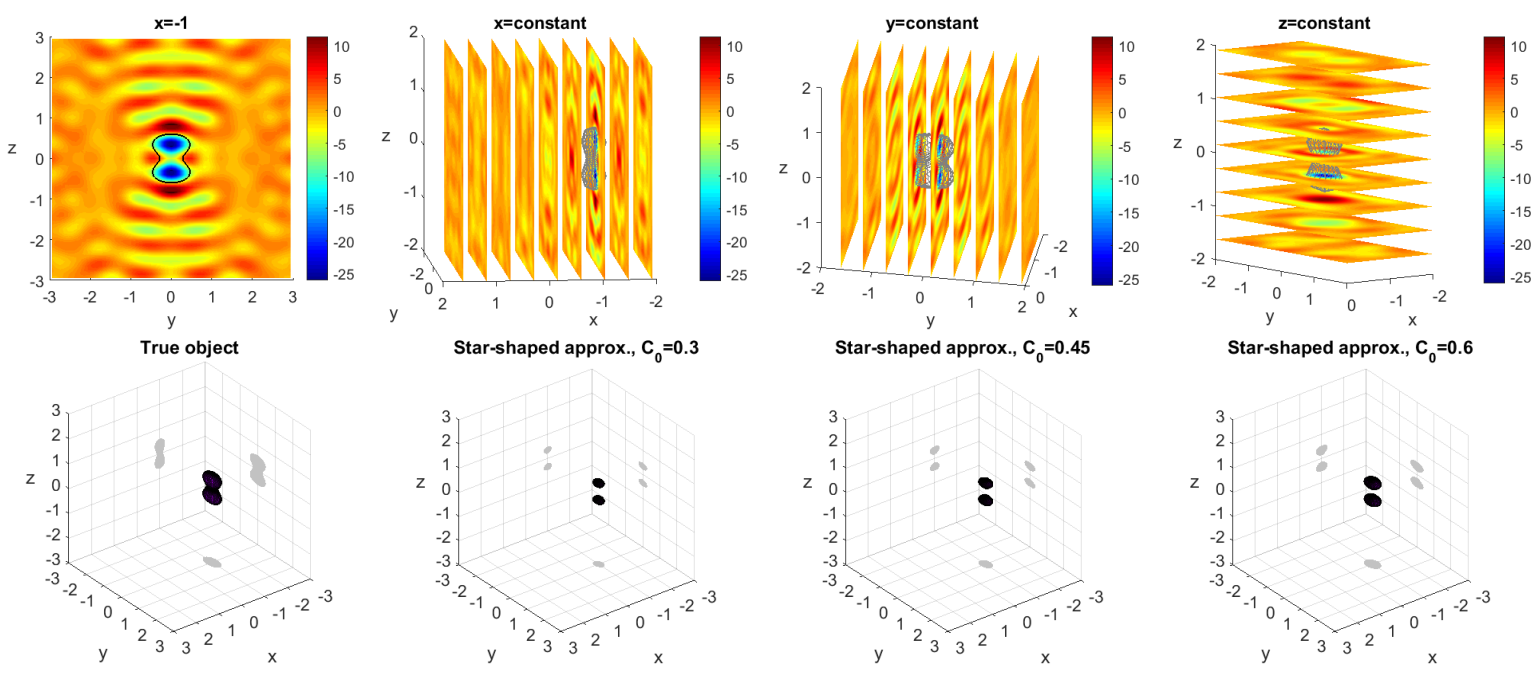

Figure 4. Counterpart of Figure 2 for $\kappa=5$.

and 5, respectively. We observe that both wavenumbers provide reconstructions with the true number of defects, correctly located and oriented. However, the method seems to have difficulties in distinguishing between shapes that are similar, and both defects seem to be ellipsoids. Nevertheless, by carefully observing the values of the TD at the plane $x=-1$ when $\kappa=5$, we realized that the largest negative values of the TD (blue colors) concentrate in a rectangular region inside the cylinder, while the region is elliptical inside the ellipsoid.

We want to illustrate now the performance of the method when dealing with more complicated geometries. To do that, we consider the configuration shown in Figure 1(c), with two nonconvex domains. The volume of the beam-like defect is bigger than the volume of the peanut-like object, and the most pronounced negative values of the TD concentrate inside the 

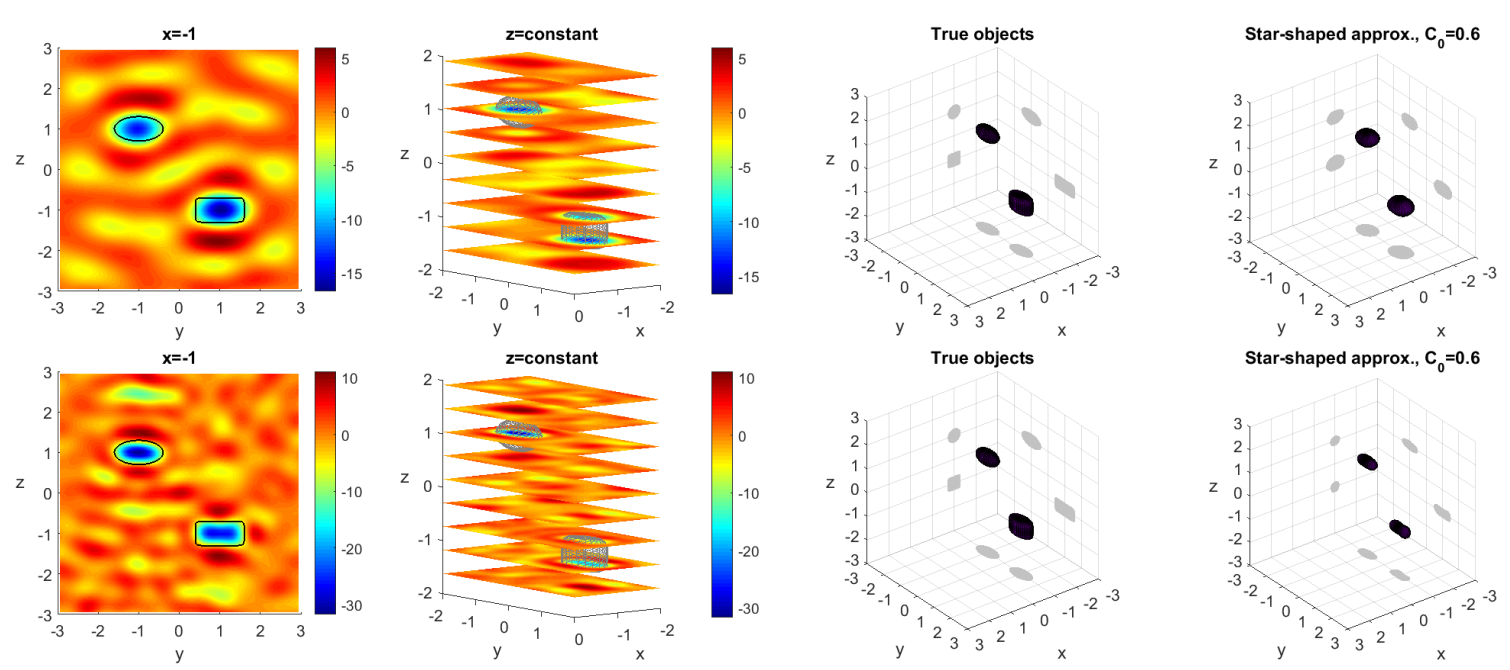

Figure 5. Counterpart of Figure 2 for $\kappa=3$ (top row) and $\kappa=5$ (bottom row) for the geometrical configuration with two convex objects.
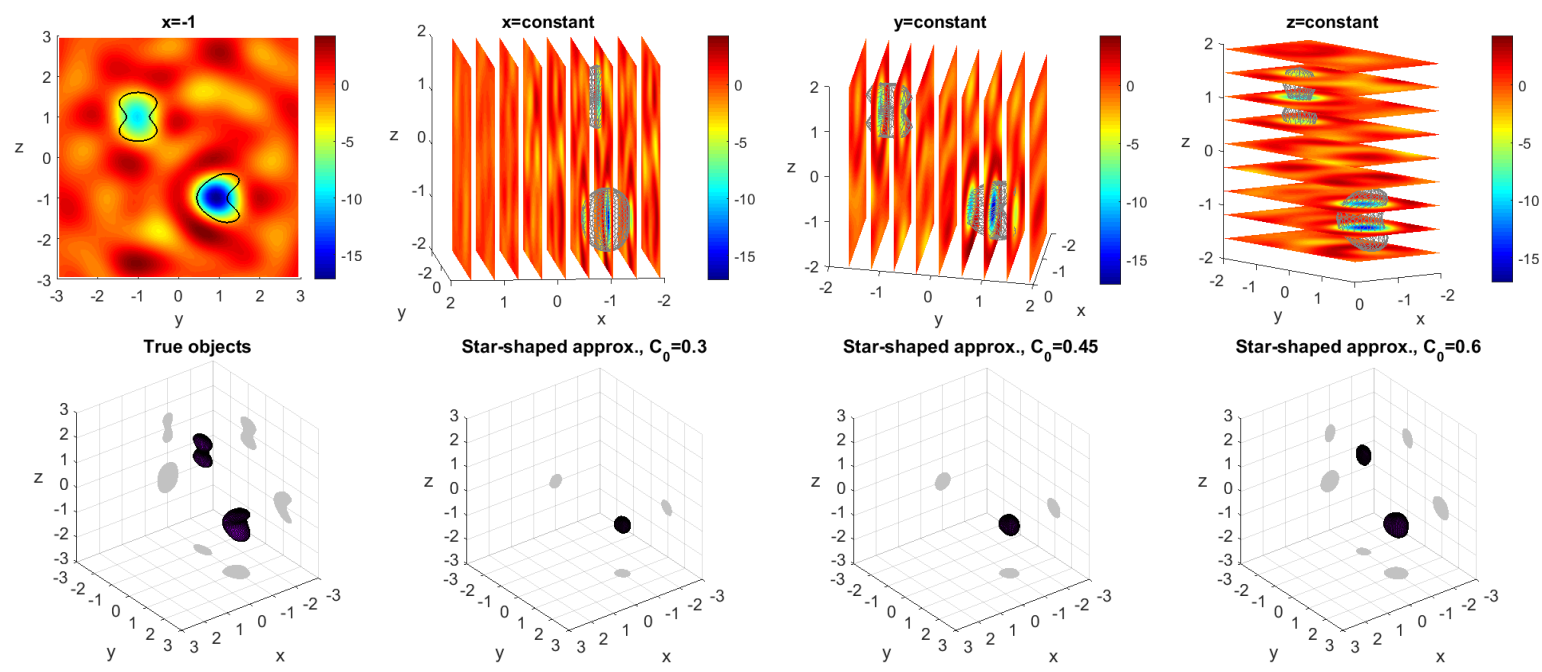

Figure 6. Counterpart of Figure 2 for $\kappa=3$ for the geometrical configuration with two nonconvex objects.

beam when $\kappa=3$ (see Figure 6 and the associated movie M111385_07.avi [local/web 2.11MB]). Only for big enough constants $C_{0}$ does our reconstruction have two objects. However, increasing the wavenumber to $\kappa=4$, the largest negative values of the TD also concentrate inside the peanut-like defect (see Figure 7 and M111385_08.avi [local/web 2.12MB]). This effect is further observed for $\kappa=5$ (see Figure 8 and M111385_09.avi [local/web 2.24MB]). Now, the number of defects is overestimated when $C_{0}=0.45$, and four defects are recovered: each true defect is approximated by two closely located ones, as observed in the first and third plots in the second row of Figure 8, which show a star- and a non-star-shaped reconstruction of them. As previously mentioned, high wavenumbers promote oscillations, which can be misin- 

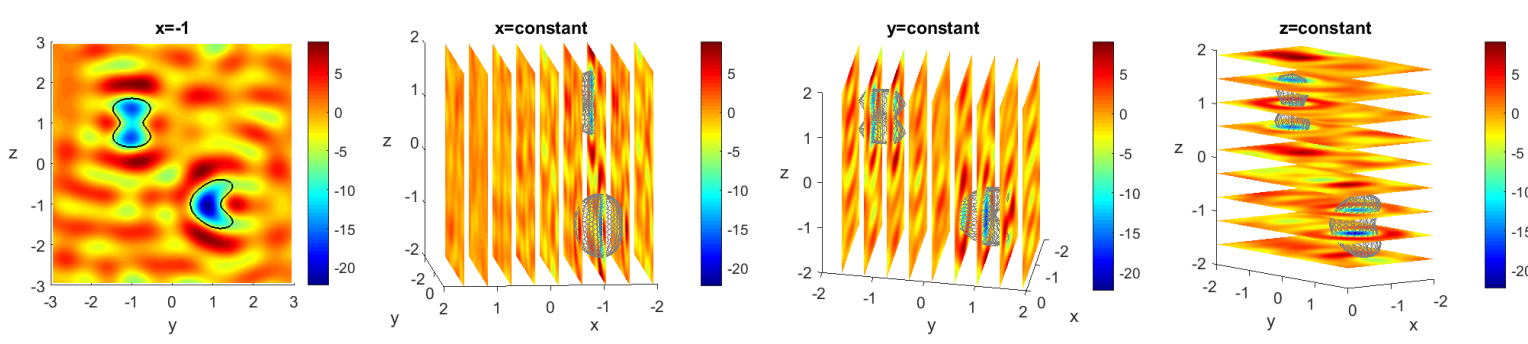

True objects
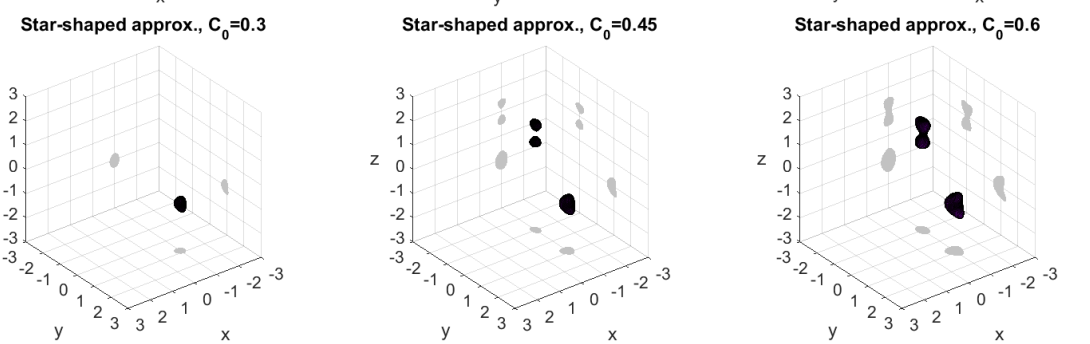

Figure 7. Counterpart of Figure 2 for $\kappa=4$ for the geometrical configuration with two nonconvex objects.
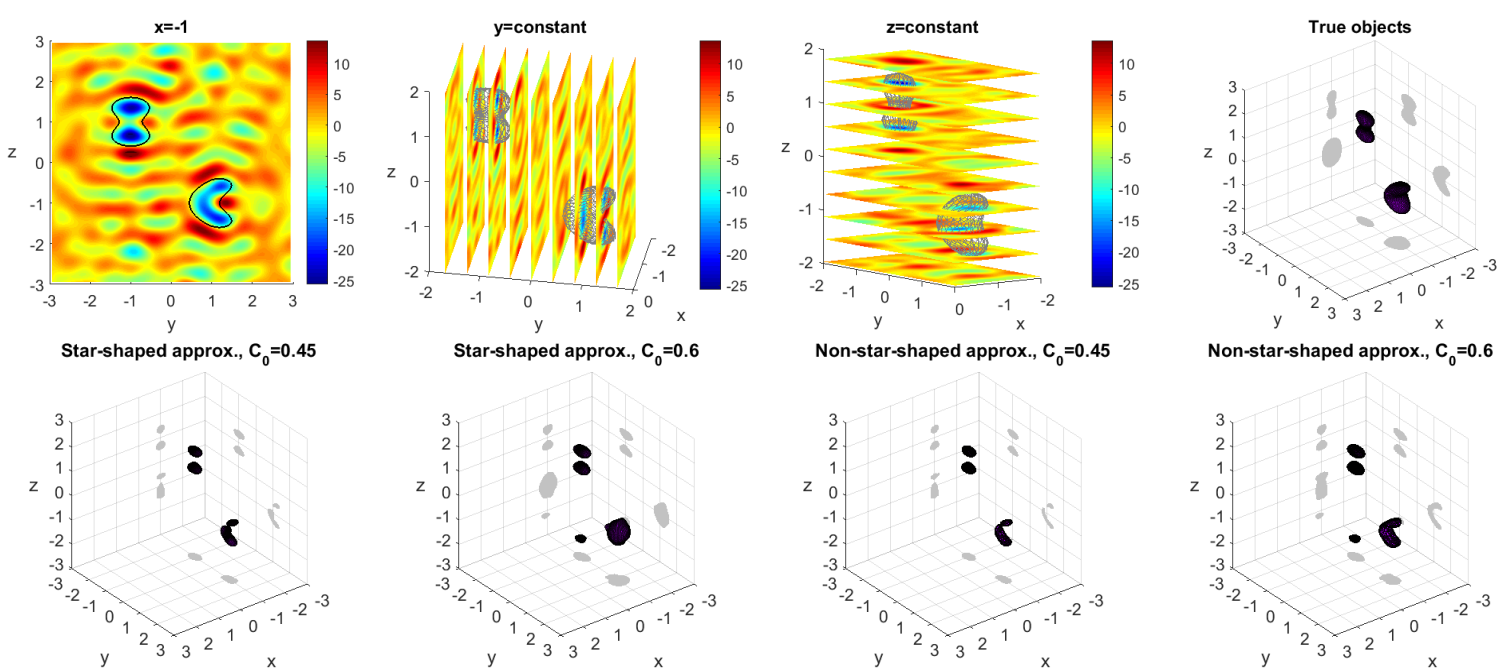

Figure 8. Counterpart of Figure 2 for $\kappa=5$ for the geometrical configuration with two nonconvex objects. Reconstructed objects are obtained by star and non-star-shaped approximations.

terpreted as small defects for big constants $C_{0}$. This effect is observed in the second and fourth plots in the second row of Figure 8, where a small spurious ellipsoidal object is found when $C_{0}=0.6$. It is also noticeable that, as expected, highly nonconvex domains could not be well approximated by star-shaped objects, as happens for the beam-like defect (see the second plot in the second row of Figure 8). We want to emphasize that this fact is not caused by the TD, which clearly identifies the true defect. By considering the non-star-shaped approximation described in section 4.2, convexity is sharply reconstructed, as observed in the last plot of the second row in Figure 8.

Repeating the experiment in a more demanding geometrical configuration with eight de- 

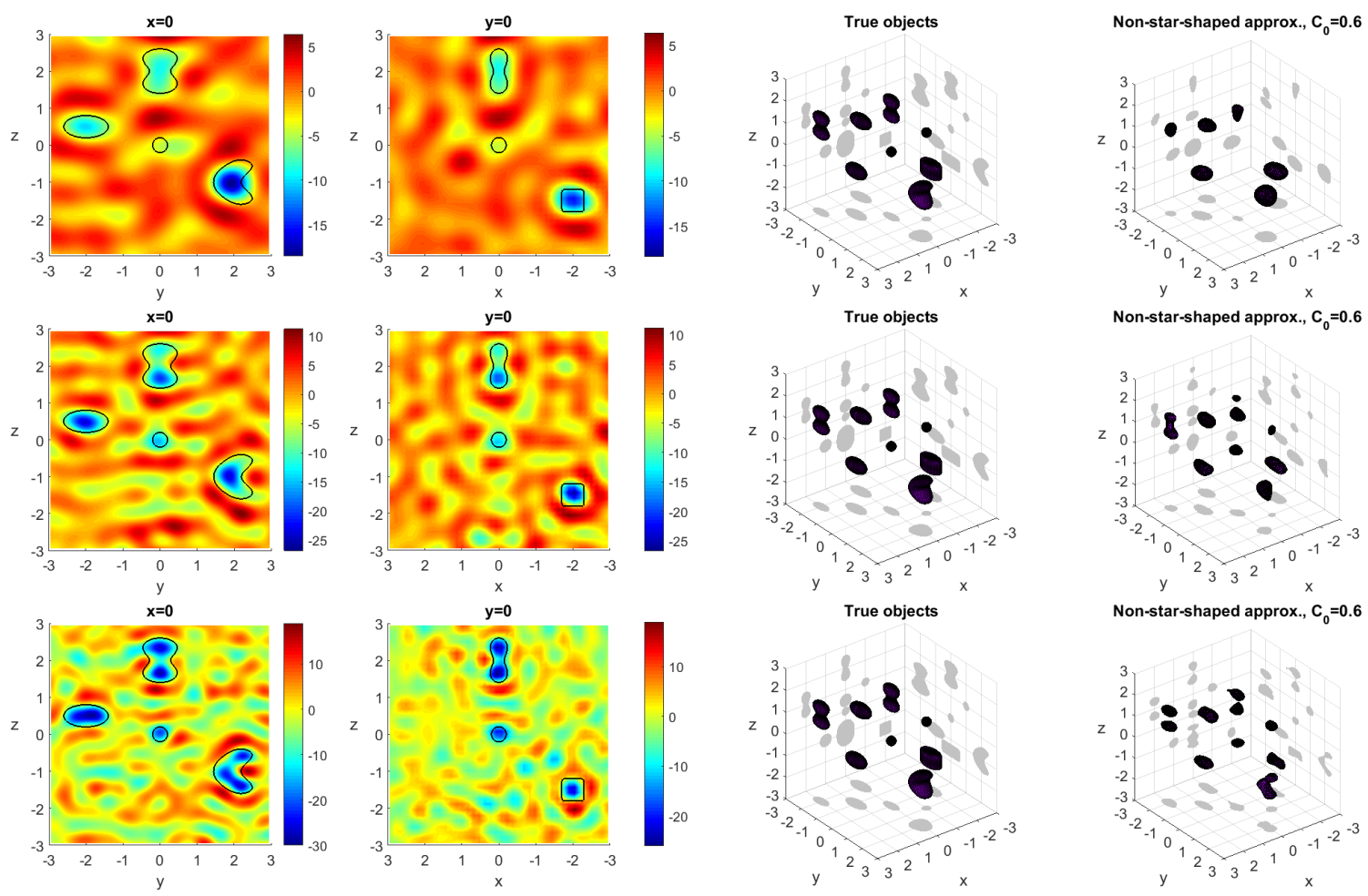

Figure 9. Counterpart of Figure 2 in a configuration with eight objects. The wavenumbers are $\kappa=3$, $\kappa=4$, and $\kappa=5$ in the first, second, and third rows, respectively.

fects (see Figure 1(d)), the method still provides very reasonable reconstructions, as can be observed in Figure 9 (see also the associated movies M111385_10.avi [local/web 2.26MB], M111385_11.avi [local/web 2.30MB], and M111385_12.avi [local/web 2.27MB]). In the first row of Figure 9 we take $\kappa=3$ and observe that only the biggest defects are captured, with a correct location and volume, but shapes are not accurately recovered. The two small spheres are missed. The wavenumber $\kappa=4$ improves the reconstruction, providing very good approximations of the correct location, size, and shape of the scatterers; the small spheres are identified now. Only one of the two peanut-like objects is split into two different objects when $C_{0}=0.6$. The reconstruction for $\kappa=5$ is qualitatively similar to that for $\kappa=4$. Summing up, by comparing the results for the three wavenumbers, we observe that, as before, a low wavenumber provides clear images where objects are clearly distinguished but shapes are not well recovered. Increasing the wavenumber, shapes are better resolved, and objects with different sizes are better captured, but the number of oscillations of the TD increases too, making it more complicated to distinguish between very small defects and oscillations. Depending on the selected constant $C_{0}$, it is feasible to include some spurious objects when the higher wavenumber is considered.

5. Conclusions. We have proposed a noniterative strategy for the detection of multiple scatterers in 3D electromagnetism that does not require a priori knowledge about the number, size, or location of the objects sought. The method relies on the computation of the 
topological derivative of a least-squares misfit functional. We have derived closed-form formulae for the topological derivative for electromagnetic cavities, perfect conductors, dielectric inclusions, and absorbing obstacles. In the end, the implementation of our method requires only evaluating fundamental solutions to the Maxwell system and their rotationals. This means that the method is straightforward to implement, and furthermore, its computational cost is almost negligible in comparison with other sampling methods. The gallery of numerical experiments shown in section 4 and in the supplementary material file M111385_01.pdf [local/web 4.66MB] demonstrate that the method is highly competitive when dealing with electromagnetic cavities, perfect conductors, and dielectric inclusions. It is able to provide sharp reconstructions in very demanding situations, where highly noisy data measured at a few observation locations (for both the near- and far-field cases) from a very reduced number of incident directions are considered. It performs very reasonably even in the case with several scatterers of different sizes and complicated shapes. However, we have observed that when dealing with absorbing obstacles, the method has difficulties in providing reasonable reconstructions when considering a reduced number of observation points and incident waves. In the numerical examples we have performed so far, it seems that it performs better for low wavenumbers than for higher ones. Our study, however, is neither exhaustive nor conclusive. A further study should be performed in this case.

Our method can be used to provide initial guesses for iterative methods, as for Newtontype algorithms. In particular, it could be of great interest for iterative methods that do not require initial guesses having the correct number of defects, such as level set methods or methods based on the computation of iterated topological derivatives. This will be the subject of our forthcoming work [36].

\section{REFERENCES}

[1] M. Abramowitz And I. A. Stegun, Handbook of mathematical functions with formulas, graphs, and mathematical tables, National Bureau of Standards Applied Mathematics Series 55, Dover, NewYork, 1964.

[2] H. Ammari, J. Garnier, V. Jugnon, And H. Kang, Stability and resolution analysis for a topological derivative based imaging functional, SIAM J. Control Optim., 50 (2012), pp. 48-76, https://doi.org/ $10.1137 / 100812501$.

[3] H. Ammari, M. S. Vogelius, And D. Volkov, Asymptotic formulas for perturbations in the electromagnetic fields due to the presence of inhomogeneities of small diameter II. The full Maxwell equations, J. Math. Pures Appl., 80 (2001), pp. 769-814, https://doi.org/10.1016/S0021-7824(01)01217-X.

[4] G. BAO, J. Lin, AND S. M. MeFIRE, Numerical reconstruction of electromagnetic inclusions in three dimensions, SIAM J. Imaging Sci., 7 (2014), pp. 558-577, https://doi.org/10.1137/130937640.

[5] A. Bendali, M. FAREs, AND J. GaY, A boundary-element solution of the Leontovitch problem, IEEE Trans. Antennas Propagation, 47 (1999), pp. 1597-1605, http://ieeexplore.iee.org/document/ $805905 /$.

[6] M. F. Ben Hassen, K. ERhard, and R. Potthast, The point-source method for $3 D$ reconstructions for the Helmholtz equation and Maxwell equations, Inverse Problems, 22 (2006), pp. 331-353, https: //doi.org/10.1088/0266-5611/22/1/018.

[7] A. Buffa, R. Hiptmair, T. von Petersdorff, and C. Schwab, Boundary element methods for Maxwell transmission problems in Lipschitz domains, Numer. Math., 95 (2003), pp. 459-485, https: //link.springer.com/article/10.1007/s00211-002-0407-z.

[8] F. CAkoni, D. Colton, And P. Monk, The Linear Sampling Method in Inverse Electromagnetic Scattering, CBMS-NSF Reg. Conf. Ser. Appl. Math. 80, SIAM, Philadelphia, 2011.

Copyright $@$ by SIAM. Unauthorized reproduction of this article is prohibited. 
[9] F. CAKoni, M. B. FAREs, And H. HAddar, Analysis of two linear sampling methods applied to electromagnetic imaging of buried objects, Inverse Problems, 22 (2006), pp. 845-867, http://iopscience.iop. org/article/10.1088/0266-5611/22/3/007.

[10] A. Carpio, T. G. Dimiduk, M. L. Rapún, and V. Selgas, Noninvasive imaging of three-dimensional micro and nanostructures by topological methods, SIAM J. Imaging Sci., 9 (2016), pp. 1324-1354, https://doi.org/10.1137/16M1068669.

[11] A. Carpio, B. T. Johansson, And M.-L. Rapún, Determining planar multiple sound-soft obstacles from scattered acoustic fields, J. Math. Imaging Vision, 36 (2010), pp. 185-199, https://link.springer. com/article/10.1007/s10851-009-0182-x.

[12] A. CARPIO AND M. L. RAPÚN, Solving inhomogeneous inverse problems by topological derivative methods, Inverse Problems, 24 (2008), 045014, http://iopscience.iop.org/article/10.1088/0266-5611/24/4/ 045014 .

[13] D. Colton, H. HAddar, And M. PiAna, The linear sampling method in inverse electromagnetic scattering theory, Inverse Problems, 19 (2003), pp. S105-S137, http://iopscience.iop.org/article/10.1088/ 0266-5611/19/6/057.

[14] D. Colton And R. Kress, Inverse Acoustic and Electromagnetic Scattering Theory, 3rd ed., Appl. Math. Sci. 93, Springer, New York, 2013.

[15] M. Costabel And F. Le Loü̈r, Shape derivatives of boundary integral operators in electromagnetic scattering. Part II: Application to scattering by a homogeneous dielectric obstacle, Integral Equations Operator Theory, 73 (2012), pp. 17-48, https://link.springer.com/article/10.1007/s00020-012-1955-y.

[16] O. Dorn And D. Lesselier, Level set methods for inverse scattering, Inverse Problems, 22 (2006), pp. R67-R131, https://doi.org/10.1088/0266-5611/22/4/R01.

[17] O. DORN AND D. LESSELIER, Level set methods for inverse scattering-Some recent developments, Inverse Problems, 25 (2009), 125001, https://doi.org/10.1088/0266-5611/25/12/125001.

[18] G. R. FEIJoo, A new method in inverse scattering based on the topological derivative, Inverse Problems, 20 (2004), pp. 1819-1840, https://doi.org/10.1088/0266-5611/20/6/008.

[19] M. Ganesh And I. G. Graham, A high-order algorithm for obstacle scattering in three dimensions, J. Comput. Phys., 198 (2004), pp. 211-242, https://doi.org/10.1016/j.jcp.2004.01.007.

[20] M. Ganesh And S. C. Hawkins, A hybrid high-order algorithm for radar cross section computations, SIAM J. Sci. Comput., 29 (2007), pp. 1217-1243, https://doi.org/10.1137/060664859.

[21] M. Ganesh and S. C. Hawkins, A high-order tangential basis algorithm for electromagnetic scattering by curved surfaces, J. Comput. Phys., 227 (2008), pp. 4543-4562, https://doi.org/10.1016/j.jcp.2008. 01.016 .

[22] M. Ganesh And S. C. Hawkins, A high-order algorithm for multiple electromagnetic scattering in three dimensions, Numer. Algorithms, 50 (2009), pp. 469-510, https://link.springer.com/article/10.1007/ s11075-008-9238-z.

[23] B. Gebauer, M. Hanke, And C. Schneider, Sampling methods for low-frequency electromagnetic imaging, Inverse Problems, 24 (2008), 015007, https://doi.org/10.1088/0266-5611/24/1/015007.

[24] B. B. Guzina AND M. Bonnet, Small-inclusion asymptotic of misfit functionals for inverse problems in acoustics, Inverse Problems, 22 (2006), pp. 1761-1785, https://doi.org/10.1088/0266-5611/22/5/014.

[25] B. B. Guzina And I. Chikichev, From imaging to material identification: A generalized concept of topological sensitivity, J. Mech. Phys. Solids, 55 (2007), pp. 245-279, https://doi.org/10.1016/j.jmps. 2006.07.009.

[26] H. HADDAR AND R. KREss, On the Fréchet derivative for obstacle scattering with an impedance boundary condition, SIAM J. Appl. Math., 65 (2004), pp. 194-208, https://doi.org/10.1137/ S0036139903435413.

[27] H. Harbrecht AND T. Hohage, Fast methods for three-dimensional inverse obstacle scattering problems, J. Integral Equations Appl., 19 (2007), pp. 237-260, https://projecteuclid.org/euclid.jiea/1190905486.

[28] A. Henrot and M. Pierre, Variation et Optimisation de Formes. Une Analyse Géométrique, Math. Appl., Springer, Berlin, 2005.

[29] F. Hettlich, The domain derivative of time-harmonic electromagnetic waves at interfaces, Math. Methods Appl. Sci., 35 (2012), pp. 1681-1689, http://dx.doi.org/10.1002/mma.2548.

[30] T. Hohage, Iterative Methods in Inverse Obstacle Scattering: Regularization Theory of Linear and Nonlinear Exponentially Ill-Posed Problems, Ph.D. thesis, University of Linz, Linz, Austria, 1999.

Copyright $\odot$ by SIAM. Unauthorized reproduction of this article is prohibited. 
[31] O. IVANYShyn AND F. LE LOUËR, Material derivatives of boundary integral operators in electromagnetism and application to inverse scattering problems, Inverse Problems, 32 (2016), 095003, https://doi.org/ 10.1088/0266-5611/32/9/095003.

[32] A. KIRSCH, The factorization method for Maxwell's equations, Inverse Problems, 20 (2004), pp. S117S134, https://doi.org/10.1088/0266-5611/20/6/S08.

[33] R. KRESS, Electromagnetic waves scattering: Scattering by obstacles, in Scattering, E. R. Pike and P. C. Sabatier, eds., Academic Press, London, 2001, pp. 191-210.

[34] F. LE LOÜ̈R, Spectrally accurate numerical solution of hypersingular boundary integral equations for three-dimensional electromagnetic wave scattering problems, J. Comput. Phys., 275 (2014), pp. 662666, https://doi.org/10.1016/j.jcp.2014.07.022.

[35] F. LE LOUËR, A spectrally accurate method for the direct and inverse scattering problems by multiple $3 d$ dielectric obstacles, submitted, (2016); preprint available from http://num.math.uni-goettingen.de/ preprints/files/2013-20.pdf.

[36] F. LE LOUËR AND M.-L. RAPÚN, Topological sensitivity for solving inverse multiple scattering problems in three-dimensional electromagnetism. Part II: Iterative method, in preparation.

[37] H. LiU, A global uniqueness for formally determined inverse electromagnetic obstacle scattering, Inverse Problems, 24 (2008), 035018, https://doi.org/10.1088/0266-5611/24/3/035018.

[38] D. W. Mackowski, Analysis of radiative scattering for multiple sphere configurations, Proc. Roy. Soc. London Ser. A, 433 (1991), pp. 599-614.

[39] P. A. Martin And P. Ola, Boundary integral equations for the scattering of electromagnetic waves by a homogeneous dielectric obstacle, Proc. Roy. Soc. Edinburgh Sect. A, 123 (1993), pp. 185-208.

[40] M. Masmoudi, J. Pommier, And B. SAmet, The topological asymptotic expansion for the Maxwell equations and some applications, Inverse Problems, 21 (2005), pp. 547-564, https://doi.org/10.1088/ 0266-5611/21/2/008.

[41] P. Monk, Finite Element Methods for Maxwell's Equations, Numer. Math. Sci. Comput., Oxford University Press, New York, 2003.

[42] C. MüLlER, Foundations of the Mathematical Theory of Electromagnetic Waves, Springer, Berlin, 1969.

[43] J.-C. NÉDÉLEC, Acoustic and Electromagnetic Equations, Integral Representations for Harmonic Problems, Appl. Math. Sci. 144, Springer-Verlag, New York, 2001.

[44] A. A. Novotny, R. A. Feijoo, C. Padra, and E. Taroco, Topological sensitivity analysis, Comput. Methods Appl. Mech. Engrg., 192 (2003), pp. 803-829, https://doi.org/10.1016/S0045-7825\%2802\% 2900599-6.

[45] W. K. PARK, Topological derivatives strategy for one-step iteration imaging of arbitrary shaped thin, curve-like electromagnetic inclusions, J. Comput. Phys., 231 (2012), pp. 1426-1439, https://doi.org/ 10.1016/j.jcp.2011.10.014.

[46] W. K. PARK, Multifrequency topological derivative for approximate shape acquisition of curve-like thin electromagnetic inhomogeneities, J. Math. Anal. Appl., 404 (2013), pp. 501-518, https://doi.org/10. 1016/j.jmaa.2013.03.040.

[47] P.-O. Persson And G. Strang, A simple mesh generator in MATLAB, SIAM Rev., 46 (2004), pp. 329345, https://doi.org/10.1137/S0036144503429121.

[48] J. Pommier And B. SAmet, The topological asymptotic for the Helmholtz equation with Dirichlet condition on the boundary of an arbitrarily shaped hole, SIAM J. Control Optim., 43 (2004), pp. 899-921, https://doi.org/10.1137/S036301290241616X.

[49] R. Potthast, Domain derivatives in electromagnetic scattering, Math. Methods Appl. Sci., 19 (1996), pp. 1157-1175.

[50] R. Potthast, Point Sources and Multipoles in Inverse Scattering Theory, Chapman \& Hall/CRC Res. Notes Math. 427, Chapman \& Hall/CRC, Boca Raton, FL, 2001.

[51] J. Sokowloski And A. Żochowski, On the topological derivative in shape optimization, SIAM J. Control Optim., 37 (1999), pp. 1251-1272, https://doi.org/10.1137/S0363012997323230.

[52] A. WAнAB, Stability and resolution analysis of topological derivative based localization of small electromagnetic inclusions, SIAM J. Imaging Sci., 8 (2015), pp. 1687-1717, https://doi.org/10.1137/141000567.

[53] A. Wahab, T. Abbas, N. Ahmed, And Q. M. Z. Zia, Detection of electromagnetic inclusions using topological sensitivity, J. Comput. Math., 35 (2017), pp. 642-671, https://doi.org/10.4208/jcm. 1609-m2016-0498. 